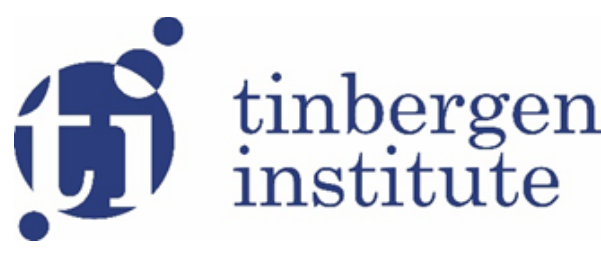

TI 2019-067/VIII

Tinbergen Institute Discussion Paper

\section{Autonomous cars and dynamic bottleneck congestion revisited: how in-vehicle activities determine aggregate travel patterns}

Xiaojuan $\mathrm{Yu}^{1}$

Vincent A.C. van den Berg ${ }^{2}$

Erik T. Verhoef

${ }^{1}$ school of Management, Huazhong University of Science and Technology, Wuhan 430074, China

${ }^{2}$ Vrije Universiteit Amsterdam 
Tinbergen Institute is the graduate school and research institute in economics of Erasmus University Rotterdam, the University of Amsterdam and VU University Amsterdam.

Contact: discussionpapers@tinbergen.nl

More TI discussion papers can be downloaded at https://www.tinbergen.nl

Tinbergen Institute has two locations:

Tinbergen Institute Amsterdam

Gustav Mahlerplein 117

1082 MS Amsterdam

The Netherlands

Tel.: +31(0)205984580

Tinbergen Institute Rotterdam

Burg. Oudlaan 50

3062 PA Rotterdam

The Netherlands

Tel.: +31(0)10408 8900 


\title{
Autonomous cars and dynamic bottleneck congestion revisited: how in-vehicle activities determine aggregate travel patterns
}

\author{
Xiaojuan $\mathrm{Yu}^{* a, b}$, Vincent A.C. van den Berg ${ }^{\mathrm{b}, \mathrm{c}}$, Erik T. Verhoef ${ }^{\mathrm{b}, \mathrm{c}}$ \\ ${ }^{a}$ School of Management, Huazhong University of Science and Technology, Wuhan 4300\%4, China \\ ${ }^{\mathrm{b}}$ Department of Spatial Economics, VU Amsterdam, De Boelelaan 1105, 1081 HV Amsterdam, The \\ Netherlands \\ ${ }^{\mathrm{c}}$ Tinbergen Institute, Gustav Mahlerplein 117, 1082 MS Amsterdam, The Netherlands
}

\begin{abstract}
We investigate the impacts of in-vehicle activities of commuters in the autonomous car on aggregate travel patterns. We allow for an autonomous car to affect the utility difference between being at home and being in the vehicle differently than the utility difference between being at work and being in the vehicle, compared to the differences experienced with a normal car. This affects the relative importance of values of travel delays, schedule delays early, and schedule delays late. Hence multiple possible changes in travel patterns may occur when autonomous cars become available. Switching to an autonomous vehicle may impose a net negative or positive externality, by raising the marginal external cost of autonomous cars themselves while lowering that of normal cars. We examine three provision regimes: marginal cost pricing, second-best pricing and profit-maximizing pricing by a private monopoly. The second-best mark-up (over marginal cost) rises with the price sensitivity, due to the increasing marginal external cost. Surprisingly, for the monopoly, mark-up may rise or fall with the price sensitivity, depending on the relative strength of the externality and of market power, where the former tends to raise it, and the latter tends to reduce it. Furthermore, the difference of the mark-up between private monopoly and second-best public provision falls as the demand becomes more price-sensitive.
\end{abstract}

Keywords: Autonomous cars; Bottleneck model; Traffic congestion; Private vs public provision.

${ }^{*}$ Corresponding author.

Email addresses: x.yu@vu.nl (Xiaojuan Yu), v.a.c.vanden.berg@vu.nl (Vincent van den Berg), e.t.verhoef@vu.nl (Erik Verhoef). 


\section{Introduction}

In recent years, car manufactures and software companies have presented prototypes of autonomous car and have announced that autonomous car will be available on the market in the near future (Fagnant and Kockelman (2015), Wadud et al. (2016)). This may provide alternative way to alleviate traffic congestion in the morning peak hours. In particular, one new and notably relevant aspect when traveling in an autonomous car is the possibility of alternative time use: time does no longer need to be invested in the driving task, but can be used for productive or recreation and leisure activities. In that way, the time in the autonomous car could become a closer substitute to time at home and/or at work. This raises an interesting and important issue: how does the in-vehicle activity (leisure or work) affect the commuters' willingness to pay and the suppliers' provision strategy for the autonomous car? Research on such behavioral impacts of autonomous driving, however, is still in its infancy.

To study these questions, we develop a model in which all commuters travel from home to work in the morning, by autonomous car or normal car. To reach their destination, they need to pass through a bottleneck, where congestion occurs. We build on the standard departure-time choice equilibrium model for a single bottleneck (Vickrey (1969), Hendrickson and Kocur (1981), Arnott et al. (1990)). That model, and its extensions, has for long been the workhorse model for studying the economics of traffic congestion (Smith (1984), Daganzo (1985), Newell (1987), Lindsey (2004), Van den Berg and Verhoef (2011a,b), Liu and Nie (2011), Li et al. (2017)).

To reflect the diverse substitution possibilities of time spent in an autonomous car, versus time spent at work or leisure, we consider the unpriced equilibrium of the bottleneck model. This setting closely follows Van den Berg and Verhoef (2016). In their setting, users' value of time falls after buying an autonomous car. We allow for the autonomous car to possibly affect the utility difference of being at home over being in the vehicle differently than the utility difference of being at work over being in the vehicle. In this way, in-vehicle activities not only result in travelers' heterogeneity in value of time, but also the value of scheduel delay. By maximizing their utility in the morning, the travelers' choice of whether to buy an autonomous car, and their departure time, can both be determined endogenously. Our model thus produces endogenous heterogeneity of drivers, if not everybody chooses to drive autonomous cars. This makes the determination of the travel pattern more complex, as it is not only determined by the value of time and schedule delay, but also by the endogenous numbers of autonomous cars and normal cars. Therefore, different in-vehicle activities give rise to different possible types of equilibrium patterns of travelers, for example in terms of the order of traveling. By analyzing the switching condition between each case, we analytically derive the conditions for each 
possible pattern to happen. While the clear distinction in various discretely different travel patterns is a natural consequence of the deterministic modeling approach, the patterns will be strongly indicative of temporal clusterings of traveler types that we may also expect to observe in reality. The same will be true for efficiency and welfare measures that we can derive.

Various studies have found that heterogeneity is important, and that heterogeneous commuters may exhibit large behavioral differences in departure time choice during peak hours, and in response to congestion policies (Arnott et al. (1988), Lindsey (2004), Van den Berg and Verhoef (2011a,b), Silva et al. (2016)). With heterogeneous preferences, drivers self-select their departure times. With queuing, the users with the higher ratio of value of schedule delay and value of time travel in the center of peak when schedule delays are small; the users with lower ratio of value of schedule delay and value of time travel in the shoulder of the peak and enjoy short travel delays in exchange for a less convenient arrival time (Arnott et al. (1990)). In Van den Berg and Verhoef (2016), the lower value of time after buying the autonomous car makes the autonomous car drivers travel in the center of the bottleneck and the normal car drivers in the shoulder. When considering different possible in-vehicle activities, various possibilities for impacts on values of time and schedule delay arise, and this leads to the possibility of different types of travel patterns arising.

Autonomous vehicles receive growing attention in policy circles and have induced extensive research interest in their impacts, operation and implications for regulation. Previous studies have for example focused on road safety (Jamson et al. (2013), de Almeida Correia and van Arem (2016), Katrakazas et al. (2015)), traffic flow (Varaiya (1993), Fajardo et al. (2011), Mahmassani (2016), de Oliveira (2017)), intersection control (Le Vine et al. (2015), Yang and Monterola (2016)), emissions (Greenblatt and Saxena (2015), Mersky and Samaras (2016)), shared autonomous vehicles operations (Ma et al. (2017), Lamotte et al. (2017), Chen et al. (2016a), Chen et al. (2016b)), and parking issues for autonomous vehicles and associated behavior patterns (Zakharenko (2016), Liu (2018), Nourinejad et al. (2018)). In terms of commuters' departure time and car type decisions, Lamotte et al. (2017) use the bottleneck model to consider separate roads for normal and autonomous cars, assuming that autonomous cars cooperate by having a departure rate equal to the capacity of their road. In their setting, autonomous cars do not affect preferences. Levin and Boyles (2016) study route choice equilibrium with mixed autonomous and normal cars in a cell transmission model, but the choices of departure time and car type kept exogenously fixed. Van den Berg and Verhoef (2016) use the bottleneck model to investigate different provision regimes of autonomous cars; considering the effects on capacity, value of time and preference heterogeneity. However, possible changes in values of schedule delay are not considered, and overall demand is kept fixed. 
From an economics standpoint, one may expect impacts on total car ownership if a new alternative is offered, and is sufficiently attractive to indeed cover a part of the total market. For congested infrastructure this is not necessarily exclusively good news, and a proper evaluation of welfare effects should take this into consideration. It is thus of great importance to incorporate price-sensitive demand when analyzing the supply strategy. To endogenously decide the optimal share and pricing of autonomous car, we follow Van den Berg and Verhoef (2016), and consider three "market schemes": marginal cost pricing, second-best public (welfare-maximizing) pricing, and private monopolistic provision. We take the interactions between the possible travel patterns and users' willingness to pay for the autonomous car into account, which raises the difficulty of modeling the suppliers' pricing decision.

In light of the above, this paper studies autonomous cars and dynamic bottleneck congestion with endogenous heterogeneity of drivers. This paper makes three main contributions to the literature. First, we regard the time spent in the autonomous car as close substitute to either time at home or time at work, and thus investigate how in-vehicle activities affect equilibrium travel patterns when automated cars have become available on the market. Second, the properties of the optimal pricing and share of autonomous cars under different provision schemes are analytically explored and compared, particularly for the interior solutions. We will find that, and explain why, the provision strategy heavily depends on travel preferences. Third, we examine the effects of price sensitivity on the provision strategy and commuters' scheduling patterns. The relative performance of the perfect competition and private monopoly in terms of the relative efficiency are also evaluated, accounting for the impact of demand elasticity.

The remainder of the paper is organized as follows. Section 2 presents the basic model set-up. Section 3 explores the equilibrium dynamic travel pattern with mixed normal car and autonomous car drivers. In Section 4, we discuss the supply scheme under fixed demand, and next extend to price-sensitive demand in Section 5. Numerical illustrations are presented in Section 6. Section 7 concludes.

\section{Model set-up}

We consider a morning commuting schedule that consists of two desired activities (i.e. being at home and being at work) and one trip between these (i.e. the journey from home to work), the timing of which is depicted in Fig.1. Every morning, $N$ commuters travel from home to a workplace connected by a single road that is subject to bottleneck congestion. Commuters obtain utility from being at home, being in the vehicle and being at work. We define a clock time $t_{S}$ as the morning start time and a 
clock time $t_{E}$ as the morning end time. Both are arbitrarily chosen, but such that all travel takes place within the interval $\left[t_{S}, t_{E}\right]$. The time in the vehicle includes the free-flow travel time and the time in the queue.

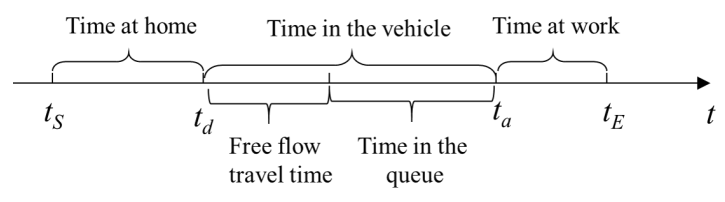

Fig. 1. An example of the morning commuting schedule.

Commuters choose their departure times to maximize their own utility, which are based on the trade-off between the utilities of activities performed at home and at work, and the journey time from home to work. Let $h[t], v(t)$ and $w[t]$ denote the utility obtained from at home, in the vehicle and at work, respectively. The commuters' utilities can be represented as:

$$
\begin{aligned}
u\left[t_{d}, t_{a}\right] & =\int_{t_{S}}^{t_{d}} h[t] d t+\int_{t_{d}}^{t_{a}} v[t] d t+\int_{t_{a}}^{t_{E}} w[t] d t \\
& =\int_{t_{S}}^{t_{d}} h[t] d t+\int_{t_{S}}^{t_{E}} v[t] d t-\int_{t_{S}}^{t_{d}} v[t] d t-\int_{t_{a}}^{t_{E}} v[t] d t+\int_{t_{a}}^{t_{E}} w[t] d t \\
& =\int_{t_{S}}^{t_{E}} v[t] d t+\int_{t_{S}}^{t_{d}}(h[t]-v[t]) d t+\int_{t_{a}}^{t_{E}}(w[t]-v[t]) d t
\end{aligned}
$$

where $t_{d}$ is the departure time and $t_{a}$ is the arrival time.

The first term in Eq.(1) is constant and unaffected by behavior, while the second and third terms involve only differences in marginal utilities. Hence, we could normalize $v[t]$ to zero in the present set-up, following Tseng and Verhoef (2008). Because later, $v[t]$ will depend on vehicle technology, we will however not make that normalization here.

To motivate making a trip, at some time point the value of $w[t]-v[t]$ must exceed the value of $h[t]-v[t]$. We assume that $h[t]-v[t]$ is non-increasing and $w[t]-v[t]$ non-decreasing over the peak, and that a time $t^{*}$ exists such that $h[t]-v[t]>w[t]-v[t]$ for $t<t^{*}$ and $h[t]-v[t]<w[t]-v[t]$ for $t>t^{*}$. With a given free flow travel time $T_{f}$, the individual would prefer to depart and arrive at moments $t_{d}^{* *}$ and $t_{a}^{* *}$ such that $h\left(t_{d}^{* *}\right)-v\left(t_{d}^{* *}\right)=w\left(t_{a}^{* *}\right)-v\left(t_{a}^{* *}\right)$ while $t_{a}^{* *}-t_{d}^{* *}=T_{f}$ is satisfied. Tseng and Verhoef (2008) called this $t_{a}^{* *}$ the ideal arrival time. Under these assumptions, travelers thus prefer to be at home until $t_{d}^{* *}$.

In accordance with Tseng and Verhoef (2008), the travel costs for someone who departs at $t_{d}$ and 
arrives at $t_{a}$ are

$$
c\left[t_{d}, t_{a}\right]=\int_{t_{d}}^{t_{a}}(h[t]-v[t]) d t+ \begin{cases}\int_{t_{a}}^{t^{* *}}((h[t]-v[t])-(w[t]-v[t])) d t & \text { if } t_{a}<t^{* *} \\ \int_{t_{a}}^{t^{* *}}((w[t]-v[t])-(h[t]-v[t])) d t & \text { if } t_{a} \geq t^{* *} .\end{cases}
$$

The conventional linear (" $\alpha-\beta-\gamma ")$ model can be written as

$$
\begin{aligned}
h[t]-v[t] & =\alpha, \\
(h[t]-v[t])-(w[t]-v[t]) & =\beta, \quad \text { for } t<t^{*}, \\
(w[t]-v[t])-(h[t]-v[t]) & =\gamma, \quad \text { for } t>t^{*} .
\end{aligned}
$$

Substituting them into Eq.(2) yields the travel cost in the traditional Vickrey's bottleneck model. While various utility specifications for $h[t]-v[t]$ and $w[t]-v[t]$ are possible, we use this linear one, to stay as close as possible to the conventional linear scheduling model which assumes constancy of $\alpha, \beta$ and $\gamma$.

People adopting an autonomous car can spend time on other activities in the car besides driving, such as professional activities like at work, or relaxing like at home. We allow for the autonomous car to affect the utility difference of being at home over being in the vehicle differently than the utility difference of being at work over being in the vehicle. This reflects possibly diverse substitution possibility of time spent in an autonomous car versus being at work or at home. The natural assumption to make is then that a switch to an autonomous car shifts $h[t]-v[t]$ downwards by an amount of $\theta_{H}$ and $w[t]-v[t]$ by an amount of $\theta_{W}$. Hence, in terms of the $\alpha-\beta-\gamma$ specification, an autonomous car decreases $\alpha$ by $\theta_{H}, \beta$ by $\theta_{H}-\theta_{W}$, and $\gamma$ by $\theta_{W}-\theta_{H}$. If the autonomous car is a closer substitute for being at home than at work, then $\theta_{H}>\theta_{W}$; and vice versa. As is shown in Fig.2, our specification leaves the ideal arrival time $t^{*}$ unchanged, as it arises from the optimal travel moment if travel took no time, and we therefore can normalize $t^{*}$ to zero.

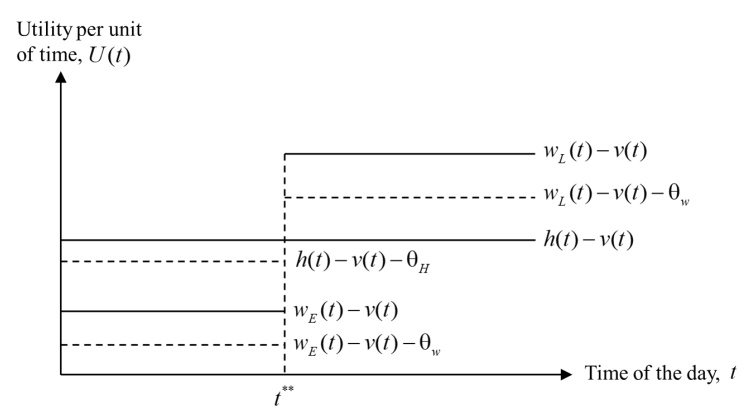

Fig. 2. Effects of in-vehicle activities. 
According to Eqs.(2-3), the travel cost of departing at time $t$ is

$$
\begin{aligned}
& c_{n}[t]=\operatorname{Max}(-\beta t, \gamma t)+\alpha T[t] \quad \text { with normal car }, \\
& c_{a}[t]=\operatorname{Max}\left(-\beta^{\prime} t, \gamma^{\prime} t\right)+\alpha^{\prime} T[t] \quad \text { with autonomous car }
\end{aligned}
$$

where $T[t]$ is the travel time experienced by the commuters departing from home at time $t$, which includes the queuing delay at the bottleneck and the free flow travel time $T_{f}$. The subscript 'n' denotes the normal car and 'a' denotes the autonomous car. The $\alpha$ is the value of time in a normal car; $\beta$ and $\gamma$ are the penalties for early and late arrivals when the commuters are driving a normal car. We use $\alpha^{\prime}, \beta^{\prime}$ and $\gamma^{\prime}$ to denote the value of time, schedule delay early and schedule delay late, for someone who owns an autonomous car. As explained, we assume that $\alpha^{\prime}=\alpha-\theta_{H}, \beta^{\prime}=\beta-\theta_{H}+\theta_{W}$, $\gamma^{\prime}=\gamma-\theta_{W}+\theta_{H}$. It is furthermore assumed that $\gamma>\alpha>\beta, \theta_{H}<\alpha, \theta_{W}-\theta_{H}<\beta$ and $\theta_{H}-\theta_{W}<\gamma$. $\gamma>\alpha>\beta$ is a standard assumption in the literature, and is consistent with many empirical evidence; the inequality $\alpha>\beta$ is needed to have an equilibrium without mass departures. The inequality $\theta_{H}<\alpha$ measures that the VOT is also positive for autonomous cars, and $\theta_{W}-\theta_{H}<\beta$ and $\theta_{H}-\theta_{W}<\gamma$ means the substitution effects are such that leisure is always more valuable at home than in the car, and work is more attractive at the job location than in the car.

The scheduling utility of a driver arriving at time $t$ is the difference between the ideal utility level, when she departed and arrived at the ideal times $t^{*}$, and the travel cost she actually incurs. Substituting Eqs.(3) into Eq.(1), we obtain the utility level when arriving at time $t$,

$$
\begin{aligned}
& u_{n}[t]=\int_{t_{S}}^{t_{E}} v[t] d t-\alpha t_{S}+(\alpha+\gamma) t_{E}-\alpha T_{f}-c_{n}[t], \\
& u_{a}[t]=\int_{t_{S}}^{t_{E}} v[t] d t-\alpha t_{S}+(\alpha+\gamma) t_{E}-\alpha^{\prime} T_{f}-c_{a}[t],
\end{aligned}
$$

where the first three terms are the ideal utility one can obtain when $T_{f}$ is zero. We normalize these first three terms to zero. The fourth term is the free-flow travel time cost. The scheduling model for the morning commute in Eq.(1) is therefore transformed into minimizing the travel cost by choosing the arrival time at work.

Both autonomous and normal car drivers choose their departure time to maximize their utility. In the dynamic equilibrium, for both types of cars, the travel costs need to be constant over the arrival times used, and can be no lower at other times. Therefore, the equilibrium utility for normal car drivers and autonomous car drivers are:

$$
\begin{aligned}
& u_{n}=-\alpha T_{f}-c_{n} ; \\
& u_{a}=-\alpha^{\prime} T_{f}-c_{a} .
\end{aligned}
$$




\section{Commuting equilibrium with mixed drivers}

We now investigate the commuting equilibrium with autonomous car and normal car drivers. The five subsections hereinafter look at five aspects of interest, namely equilibrium travel patterns, properties of the equilibrium travel patterns, users' travel cost, marginal willingness to pay for the autonomous car, and marginal social benefit.

\subsection{Equilibrium travel patterns}

To keep the travel costs constant over time before $t^{*}$, the travel time must grow linearly with arrival time at a rate $\beta / \alpha$ for drivers of normal cars, so that the decrease in schedule delay cost over arrival time is matched by the increase in the queuing cost. Similarly, after $t^{*}$, the travel time must shrink at a rate $\gamma / \alpha$ with arrival time. Now the travel delay of normal car drivers have slopes $\beta / \alpha$ and $\gamma / \alpha$, while those of autonomous car drivers naturally have slopes $\beta^{\prime} / \alpha^{\prime}$ and $\gamma^{\prime} / \alpha^{\prime}$. When both car types are present, autonomous car and normal car drivers travel separately and self-select into the center peak or the shoulder of the peak, depending on the equilibrium slopes of the travel delay functions just described. Increased ratios $\beta^{\prime} / \alpha^{\prime}$ and $\gamma^{\prime} / \alpha^{\prime}$ (relative to $\beta / \alpha$ and $\gamma / \alpha$ ) mean that the autonomous car drivers can enjoy the lower schedule delays in the center peak as they care less about travel times than normals car drivers do; and vice versa for decreased ratios. With the formulation in Section 2, we can derive different possible dynamic departure/arrival patterns at user equilibrium, when treating ownership of autonomous cars as given.

As is shown in Fig.3, five possible departure/arrival patterns can in fact appear. The travel delay in Fig.3 can be interpreted as iso-cost lines, with travel delay plotted along the vertical axis and schedule delay along the horizontal axis. The dashed lines denote an out-of-equilibrium continuation of the iso-cost functions for the corresponding case. They indicate what the travel times would have to be, but are not, for users to be willing to travel at those moments, outside their own equilibrium time window. The entire population can thus be subdivided into four groups: autonomous and normal car drivers, who can both be further subdivided into groups arriving before or after $t^{*}$. For a certain schedule preference, the equilibrium travel pattern are divided into two cases according to the share of autonomous car owners, i.e. high-share and low-share cases. We use "H" and "L" to denote these two cases. ${ }^{1}$ In Cases 1H, 1L, 2H and 2L, only three out of the four groups exist; while in Case 3, all

\footnotetext{
${ }^{1}$ Under the assumption of $\gamma>\alpha>\beta$, we can easily prove that it is impossible for $\beta^{\prime} / \alpha^{\prime}<\beta / \alpha$ and $\gamma^{\prime} / \alpha^{\prime}<\gamma / \alpha$ happening simultaneously, where the normal car drivers travel in the peak and autonomous car drivers travel in the shoulder. This case can be ignored.
} 


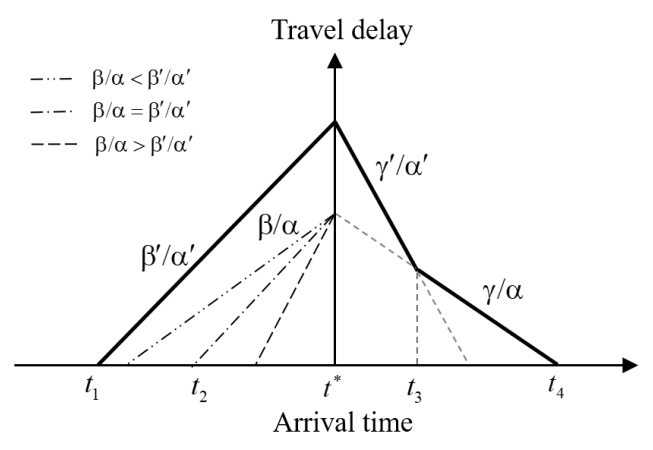

(a) Case $1 \mathrm{H}$

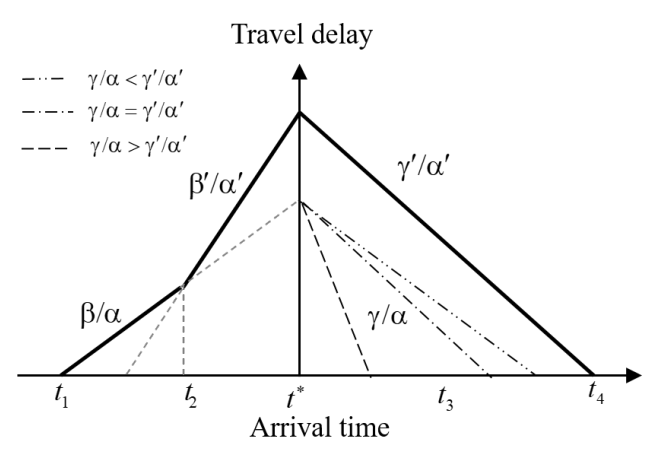

(c) Case $2 \mathrm{H}$

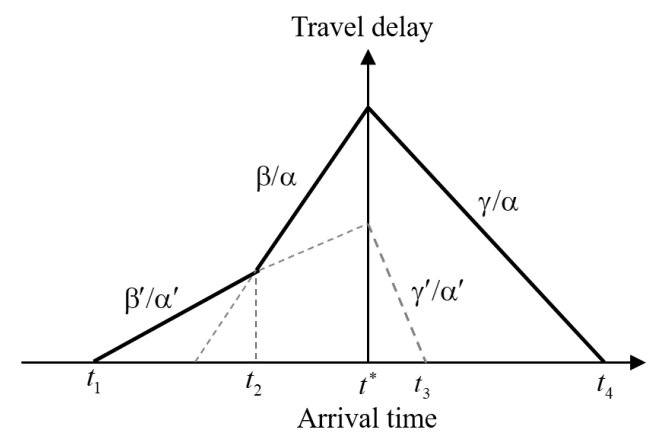

(b) Case 1L

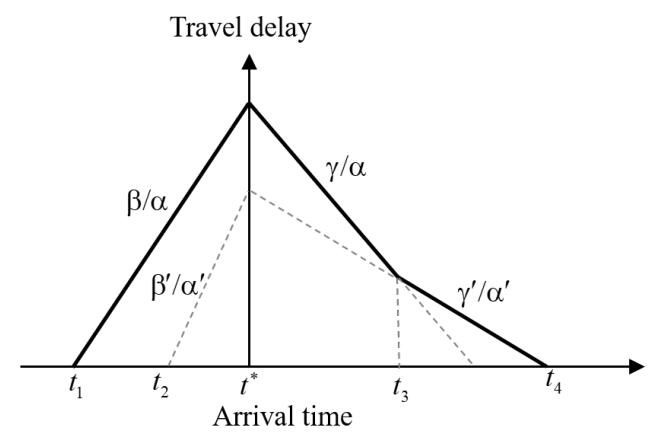

(d) Case 2L

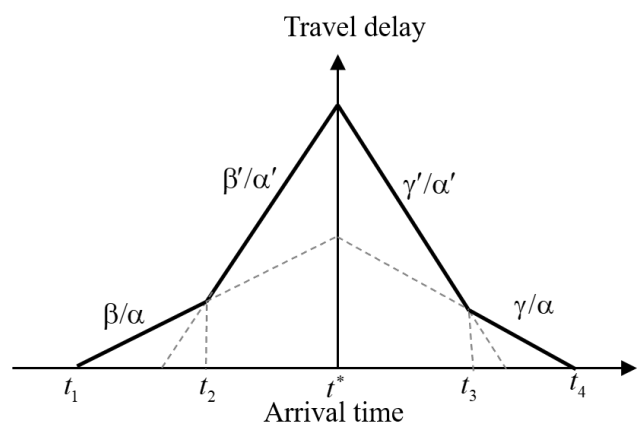

(e) Case 3

Fig. 3. Possible travel patterns with mixed drivers.

Note: In Case $1 \mathrm{H}$, there are three conditions according to the relationship between $\beta / \alpha$ and $\beta^{\prime} / \alpha^{\prime}$; in Case $2 \mathrm{H}$, there are three conditions according to the relationship between $\gamma / \alpha$ and $\gamma^{\prime} / \alpha^{\prime}$. 
four groups are present. In addition, in Case $1 \mathrm{H}$ and Case $2 \mathrm{H}$, the slope of the queuing delay, more specifically, $\beta^{\prime} / \alpha^{\prime}$ (in Case $1 \mathrm{H}$ ) and $\gamma^{\prime} / \alpha^{\prime}$ (in Case $2 \mathrm{H}$ ), can be higher or lower than $\beta / \alpha$ and $\gamma / \alpha$. But in Cases 1L, 2L and 3, the ordering of slopes of the travel delay between autonomous cars and normal cars is uniquely determined. Van den Berg and Verhoef (2016) had only an effect on the value of time, so their setting is a special case of Case 3 .

\subsection{Properties of the equilibrium travel patterns}

The travel patterns are jointly determined by the heterogeneity effects and the share of autonomous cars. The heterogeneity effects determine the arrival orders of the two types of cars, as shown in the second column in Table 1 , where $t_{1}, t_{2}, t_{3}, t_{4}$ and $t^{*}$ are defined in Fig.3. The share of autonomous cars determines whether the drivers will arrive before or after $t^{*}$, which is solved from the critical time points of each case, as specified in the third column. Combining the above two effects, we can summarize the conditions for each equilibrium travel pattern to happen in more detail, as given in the last two columns in Table 1. The derivations are provided in Appendix A. We call the critical switching shares between cases $\mathrm{H}$ and $\mathrm{L}$ as "critical share" $F_{i j}^{\#}$ and compactly denote them as $F^{\#}$ later.

\section{Table 1}

The conditions for each pattern to happen.

\begin{tabular}{|c|c|c|c|c|c|}
\hline Equilibrium & Preference conditions & Time conditions & Res & sult & \\
\hline Case $1 \mathrm{H}$ & $\gamma^{\prime} / \alpha^{\prime}>\gamma / \alpha$ & $t_{3}>t^{*}, t_{2}>t_{1}$ & $\begin{array}{l}\theta_{H} / \theta_{W}>\alpha /(\alpha-\beta) \\
1<\theta_{H} / \theta_{W}<\alpha /(\alpha-\beta)\end{array}$ & $\begin{array}{l}\& \\
\&\end{array}$ & $\begin{array}{l}F_{11}^{\#}\left(\theta_{H}, \theta_{W}\right)<f<1 \\
F_{31}^{\#}\left(\theta_{H}, \theta_{W}\right)<f<1\end{array}$ \\
\hline Case 1L & $\beta / \alpha>\beta^{\prime} / \alpha^{\prime}, \gamma^{\prime} / \alpha^{\prime}>\gamma / \alpha$ & $t_{2}<t^{*}$ & $\theta_{H} / \theta_{W}>\alpha /(\alpha-\beta)$ & $\&$ & $f<F_{11}^{\#}\left(\theta_{H}, \theta_{W}\right)$ \\
\hline Case $2 \mathrm{H}$ & $\beta^{\prime} / \alpha^{\prime}>\beta / \alpha$ & $t_{2}<t^{*}, t_{4}>t_{3}$ & $\begin{array}{l}\theta_{H} / \theta_{W}<\alpha /(\alpha+\gamma) \\
\alpha /(\alpha+\gamma)<\theta_{H} / \theta_{W}<1\end{array}$ & $\begin{array}{l}\& \\
\&\end{array}$ & $\begin{array}{l}F_{22}^{\#}\left(\theta_{H}, \theta_{W}\right)<f<1 \\
F_{32}^{\#}\left(\theta_{H}, \theta_{W}\right)<f<1\end{array}$ \\
\hline Case 2L & $\beta^{\prime} / \alpha^{\prime}>\beta / \alpha, \gamma^{\prime} / \alpha^{\prime}<\gamma / \alpha$ & $t_{3}>t^{*}$ & $\theta_{H} / \theta_{W}<\alpha /(\alpha+\gamma)$ & $\&$ & $f<F_{22}^{\#}\left(\theta_{H}, \theta_{W}\right)$ \\
\hline Case 3 & $\beta^{\prime} / \alpha^{\prime}>\beta / \alpha, \gamma^{\prime} / \alpha^{\prime}>\gamma / \alpha$ & $t_{2}>t_{1}, t_{4}>t_{3}$ & $\begin{array}{l}\alpha /(\alpha+\gamma)<\theta_{H} / \theta_{W}<1 \\
1<\theta_{H} / \theta_{W}<\alpha /(\alpha-\beta)\end{array}$ & & $\begin{array}{l}f<F_{32}^{\#}\left(\theta_{H}, \theta_{W}\right) \\
f<F_{31}^{\#}\left(\theta_{H}, \theta_{W}\right)\end{array}$ \\
\hline $\begin{array}{l}\text { Note: } F_{11}^{\#}\left(\theta_{1}\right. \\
\frac{1}{1+\frac{\alpha}{\gamma}\left(1-\frac{\theta_{W}}{\theta_{H}}\right)},\end{array}$ & $\begin{array}{l})=\frac{1}{1+\frac{\beta^{\prime} / \alpha^{\prime}}{\gamma / \alpha}}=\frac{1}{\left.1+\frac{\alpha\left(\beta-\theta H^{+}+\theta_{W}\right)}{\gamma(\alpha-\theta H}\right)} \\
\left.H, \theta_{W}\right)=\frac{1}{1+\frac{\left(\beta^{\prime}-\beta\right) /\left(\alpha^{\prime}-\alpha\right)}{\beta / \alpha}}=\frac{1+\frac{\alpha}{\beta}}{1+\frac{\alpha}{\beta}}\end{array}$ & $\begin{array}{l}F_{22}^{\#}\left(\theta_{H}, \theta_{W}\right)=\frac{1}{1+} \\
\frac{1}{\left(\frac{\theta_{W}}{\theta_{H}}-1\right)} . \text { When } 1\end{array}$ & $\begin{array}{l}\frac{\alpha^{\prime}}{\alpha}=\frac{1}{1+\frac{\alpha\left(\gamma+\theta_{H}-\theta_{W}\right)}{\beta\left(\alpha-\theta_{H}\right)}}, F_{31}^{\#}\left(\theta_{H},\right. \\
\theta_{H} / \theta_{W}<\alpha /(\alpha-\beta), F_{31}^{\#}>\end{array}$ & & $\begin{array}{l}=\frac{1}{1+\frac{\left(\gamma^{\prime}-\gamma\right) /\left(\alpha^{\prime}-\alpha\right)}{\gamma / \alpha}}= \\
\text { always exists, and }\end{array}$ \\
\hline
\end{tabular}

Table 1 shows that the equilibrium travel pattern is governed by the ratio $\theta_{H} / \theta_{W}$ and the share of users with autonomous cars $f$. Given $\theta_{H}$ and $\theta_{W}$, as $f$ increases from 0 to 1 , (1) if $\theta_{H} / \theta_{W}>\alpha /(\alpha-\beta)$, 
the travel pattern will change from Case $1 \mathrm{~L}$ to Case $1 \mathrm{H} ;$ (2) if $\theta_{H} / \theta_{W}<\alpha /(\alpha+\gamma)$, the travel pattern will change from Case $2 \mathrm{~L}$ to Case $2 \mathrm{H}$; (3) if $\alpha /(\alpha+\gamma)<\theta_{H} / \theta_{W}<1$, the travel pattern will change from Case 3 to Case 2H; (4) if $1<\theta_{H} / \theta_{W}<\alpha /(\alpha-\beta)$, the travel pattern will change from Case 3 to Case $1 \mathrm{H}$. We call the situation that is infinitely close to the switching conditions the "critical case". For instance, the critical case between case $1 \mathrm{H}$ and $1 \mathrm{~L}$ means the travel pattern is such that the autonomous cars depart before the normal cars, and all autonomous car drivers arrive early for work, while all normal car drivers arrive late for work.

\subsection{Users' travel cost}

Based on the earlier discussion we can obtain the travel cost of normal car drivers $c_{n}$ and that of autonomous car drivers $c_{a}$ in each case, which are summarized in Table 2.

Table 2

Travel costs of normal car and autonomous car in different cases in no-toll equilibrium.

\begin{tabular}{cllcc}
\hline Case & \multicolumn{1}{c}{ Normal car drivers $c_{n}$} & Autonomous car drivers $c_{a}$ & $\frac{d c_{n}}{d f}$ & $\frac{d c_{a}}{d f}$ \\
\hline Case 1H & $\frac{\gamma N}{s}-\frac{\gamma}{\beta+\gamma}\left(\gamma^{\prime} f+\frac{\gamma \alpha^{\prime}}{\alpha}(1-f)\right) \frac{N}{s}+\alpha T_{f}$ & $\frac{\beta^{\prime} \gamma}{\beta+\gamma} \frac{N}{s}\left(\frac{\alpha^{\prime}}{\alpha}(1-f)+\frac{\gamma^{\prime}}{\gamma} f\right)+\alpha^{\prime} T_{f}$ & $<0$ & $>0$ \\
Case 1L & $\frac{\gamma}{\beta+\gamma} \frac{N}{s}\left(\beta(1-f)+\frac{\alpha \beta^{\prime}}{\alpha^{\prime}} f\right)+\alpha T_{f}$ & $\frac{\beta^{\prime}}{\beta+\gamma} \frac{N}{s}\left(\gamma+\frac{\alpha^{\prime} \beta-\alpha \beta^{\prime}}{\alpha^{\prime}} f\right)+\alpha^{\prime} T_{f}$ & $<0$ & $>0$ \\
Case 2H & $\frac{\alpha^{\prime} \beta}{\beta+\gamma} \frac{N}{s}\left(\frac{\gamma^{\prime}}{\alpha^{\prime}}+(1-f)\left(\frac{\beta^{\prime}}{\alpha^{\prime}}-\frac{\beta}{\alpha}\right)\right)+\alpha T_{f}$ & $\frac{\alpha^{\prime} \gamma^{\prime}}{\beta+\gamma} \frac{N}{s}\left((1-f) \frac{\beta}{\alpha}+f \frac{\beta^{\prime}}{\alpha^{\prime}}\right)+\alpha^{\prime} T_{f}$ & $<0$ & $>0$ \\
Case 2L & $\frac{\alpha \beta}{\beta+\gamma} \frac{N}{s}\left(\frac{\gamma^{\prime}}{\alpha^{\prime}} f+\frac{\gamma}{\alpha}(1-f)\right)+\alpha T_{f}$ & $\frac{\gamma^{\prime} N}{s}-\frac{\alpha \gamma^{\prime}}{\beta+\gamma}\left(\frac{\gamma^{\prime}}{\alpha^{\prime}} \frac{f N}{s}+\frac{\gamma}{\alpha} \frac{(1-f) N}{s}\right)+\alpha^{\prime} T_{f}$ & $<0$ & $>0$ \\
Case 3 & $\frac{\delta N}{s}+\alpha T_{f}$ & $\frac{\alpha^{\prime}}{\alpha} \frac{\delta N}{s}+\frac{\gamma^{\prime} \alpha-\gamma \alpha^{\prime}}{\alpha} \frac{\left(\beta \alpha^{\prime}-\beta^{\prime} \alpha\right)}{(\beta+\gamma)\left(\alpha^{\prime}-\alpha\right)} \frac{f N}{s}+\alpha^{\prime} T_{f}$ & $=0$ & $>0$ \\
\hline
\end{tabular}

The last two columns in Table 2 show that the travel cost of normal car drivers decreases with the share of autonomous cars; only for Case 3 these costs are unaffected by it. This reflects that autonomous car drivers impose a non-negative external effect on normal car drivers. Meanwhile, the higher the share of autonomous car drivers, the larger their own travel costs. Autonomous car drivers thus impose a negative marginal externality on their own group.

Next, we can see that for every case, $c_{a}<c_{n}$ always holds. This is intuitive, as a normal car driver could adopt the autonomous car, not change the moment of driving, and benefit from the reduced disutility of being in the car rather than at home or work. Any induced change in travel moment will only bring further benefits.

\subsection{Marginal willingness to pay for the autonomous car}

Each traveler maximizes her own utility by choosing arrival time and traveling mode (i.e. type of vehicle). The marginal willingness to pay for the autonomous car, $\Delta u$, can hence be determined as 
the difference between the utility that a driver reaches over the course of the morning if one drives the autonomous car, minus the utility when driving the normal car, where the utility is defined by Eqs.(5). For a user who switches from a normal car to an autonomous car, the scheduling utility changes by:

$$
\Delta u=u_{a}-u_{n}=\theta_{H} T_{f}+c_{n}-c_{a} .
$$

As previously stated, the autonomous car reduces the travel cost. Hence the willingness to pay for the autonomous car is positive, i.e. $\Delta u>0$ always holds. In Eq.(7), this occurs because the first term is positive, and $c_{n}>c_{a}$. We can also derive that $d \Delta u / d f<0$. The larger $f$ is, the smaller the difference in the level of scheduling utility between car types, and thus the less switching raises one's own utility.

\subsection{Marginal social benefit}

The marginal social benefit of one person switching from a normal car to an autonomous car is the sum of the marginal willingness to pay of the switching driver, the marginal external benefit to all autonomous car drivers (which will be negative), and the marginal external benefits to all normal car drivers (which will be positive). The marginal external benefit to all autonomous car drivers is the difference of the derivative of their generalized travel costs with respect to the number of normal car drivers and to the number of the autonomous car drivers, multiplied by the number of autonomous car drivers; and similarly for the normal car drivers. The marginal external benefits to all autonomous car drivers, $M E B_{a}$, and to all normal car drivers, $M E B_{n}$, are therefore:

$$
M E B_{a}=-\frac{\partial c_{a}}{\partial N_{a}} N_{a}+\frac{\partial c_{a}}{\partial N_{n}} N_{a} ; \quad M E B_{n}=\frac{\partial c_{n}}{\partial N_{n}} N_{n}-\frac{\partial c_{n}}{\partial N_{a}} N_{n}
$$

where $N_{a}$ and $N_{n}$ are the numbers of autonomous car drivers and normal car drivers, respectively, which satisfy $N_{a}=f \cdot N$, and $N_{n}=(1-f) \cdot N$.

After substituting the travel cost of Table 2 into Eq.(8), we can derive the marginal external benefit to normal car and autonomous car drivers in Table 3.

Each pair of $\left(\theta_{H}, \theta_{W}\right)$ may of course apply for a high and a low share of autonomous car owners, labeled cases $\mathrm{H}$ and $\mathrm{L}$ above. The marginal social benefit conditional on having a "high" versus a "low" share of autonomous car drivers can thus be written as,

$$
\begin{aligned}
M S B_{H} & =\Delta u_{H}+M E B_{n H}+M E B_{a H} \\
M S B_{L} & =\Delta u_{L}+M E B_{n L}+M E B_{a L} .
\end{aligned}
$$




\section{Table 3}

Marginal external benefit for normal car and autonomous car drivers.

\begin{tabular}{cll}
\hline Case & Normal car $M E B_{n}$ & Autonomous car $M E B_{a}$ \\
\hline Case 1H & $\frac{\gamma^{2}}{(\beta+\gamma)}\left(\frac{\gamma^{\prime}}{\gamma}-\frac{\alpha^{\prime}}{\alpha}\right) \frac{(1-f) N}{s}>0$ & $-\frac{\beta^{\prime} \gamma}{\beta+\gamma}\left(\frac{\gamma^{\prime}}{\gamma}-\frac{\alpha^{\prime}}{\alpha}\right) \frac{f N}{s}<0$ \\
Case 1L & $-\frac{\gamma}{\beta+\gamma} \frac{\alpha \beta^{\prime}-\alpha^{\prime} \beta}{\alpha^{\prime}} \frac{(1-f) N}{s}>0$ & $-\frac{\beta^{\prime}}{\beta+\gamma} \frac{\alpha^{\prime} \beta-\alpha \beta^{\prime}}{\alpha^{\prime}} \frac{f N}{s}<0$ \\
Case 2H & $-\frac{\alpha^{\prime} \beta}{\beta+\gamma}\left(\frac{\beta}{\alpha}-\frac{\beta^{\prime}}{\alpha^{\prime}}\right) \frac{(1-f) N}{s}>0$ & $-\frac{\alpha^{\prime} \gamma^{\prime}}{\beta+\gamma}\left(\frac{\beta^{\prime}}{\alpha^{\prime}}-\frac{\beta}{\alpha}\right) \frac{f N}{s}<0$ \\
Case 2L & $-\frac{\alpha \beta}{\beta+\gamma}\left(\frac{\gamma^{\prime}}{\alpha^{\prime}}-\frac{\gamma}{\alpha}\right) \frac{(1-f) N}{s}>0$ & $\frac{\alpha \gamma}{\beta+\gamma}\left(\frac{\gamma^{\prime}}{\alpha^{\prime}}-\frac{\gamma}{\alpha}\right) \frac{f N}{s}<0$ \\
Case 3 & 0 & $\frac{\alpha^{\prime} \gamma-\alpha \gamma^{\prime}}{\alpha} \frac{\left(\beta \alpha^{\prime}-\beta^{\prime} \alpha\right)}{(\beta+\gamma)\left(\alpha^{\prime}-\alpha\right)} \frac{f N}{s}<0$ \\
\hline
\end{tabular}

\section{Provision regimes with fixed demand}

This section examines the equilibrium outcomes under three provision regimes of autonomous cars: marginal cost pricing (MC), socially optimal second-best public pricing (Pub), and pricing with private monopoly (PM). For now the total amount of travel $N$ is fixed. Section 5 will look at the price-sensitive demand. The travel patterns, of course, are endogenous and may thus vary between the regimes.

Following Van den Berg and Verhoef (2016), the generalized price per trip is the sum of the travel cost, the automobile cost and the per-trip mark-up on the car. The mark-up is determined by the producer of the car and is expressed as a per-trip equivalent. We normalize the normal cars' mark-up and automobile cost to zero. For autonomous cars, we use $M U_{a}$ to denote the mark-up per trip, and $M C_{a}$ the automobile cost. The $M U_{a}$ and $M C_{a}$ therefore reflects by how much the mark-up and marginal production cost of autonomous cars exceed those for normal cars, which may be negative, zero or positive. The generalized utility of normal car is $u_{n}$ and that of autonomous car is hence $u_{a}-M C_{a}-M U_{a}$.

\subsection{Marginal cost provision}

The provision at marginal cost means the price of an autonomous car equals marginal costs, implying a zero mark-up. It will lead to over-consumption of autonomous cars if there is a negative externality from the consumers' purchase, and to under-supply under a positive externality. ${ }^{2}$ The user equilibrium can be expressed as:

$$
\begin{gathered}
\Delta u_{H}(f, N)=M C_{a}, \quad \text { if } F^{\#}\left(\theta_{H}, \theta_{W}\right) \leq f \leq 1, \\
\Delta u_{L}(f, N)=M C_{a}, \quad \text { if } \quad 0 \leq f \leq F^{\#}\left(\theta_{H}, \theta_{W}\right),
\end{gathered}
$$

\footnotetext{
${ }^{2}$ In this Section, we do not consider road pricing, and congestion is not internalized through tolls.
} 
where $\Delta u_{H}$ and $\Delta u_{L}$ are the marginal willingness to pay with high share and low share case, respectively. The $M C_{a}$ is the excess per-peak cost of owning an autonomous vehicle.

In equilibrium, the share of autonomous cars can be over or below the critical share $F^{\#}$, that defines in Table 1, whether Case $\mathrm{H}$ or Case L will apply. To find the marginal cost that distinguishes the high and low share cases, we compare the equilibrium share and the critical share $F^{\#}$. A smaller marginal cost naturally leads to a higher share of autonomous cars. Apart from the interior solutions, however, often, there are also corner solutions. We summarize the equilibrium share of all cases in Appendix C.

\subsection{Second-best public provision}

Second-best public provision is assumed to maximize the total social welfare, $S W$, under the constraint that the bottleneck remains untolled. Total social welfare is the integral of marginal social benefits, given by Eq.(9), minus the automobile cost of autonomous cars. The optimization problem depends on whether $f$ exceeds $F^{\#}$ :

$$
\max _{f, M U_{a}} S W=\max _{f, M U_{a}}\left\{\begin{array}{l}
\int_{0}^{F^{\#}} M S B_{L}(f) \cdot N d f+\int_{F^{\#}}^{f} M S B_{H}(f) \cdot N d f-M C_{a} \cdot f N, \quad \text { if } F^{\#} \leq f \leq 1 \\
\int_{0}^{f} M S B_{L}(f) \cdot N d f-M C_{a} \cdot f N, \quad \text { if } 0 \leq f \leq F^{\#}
\end{array}\right.
$$

Eq.(11) shows that to reach the social optimum, a public provider should set a price that equates the marginal social costs and marginal cost of autonomous cars. Combining this with the user equilibrium that the willingness to pay should equal the sum of the marginal cost and the mark-up for autonomous cars, we find

$$
M U_{a}=\frac{d c_{a}}{d f}+\frac{d c_{n}}{d f}(1-f)=-M E B_{a}-M E B_{n}
$$

implying that the mark-up under second-best public supply is the sum of the negative marginal external benefit to all autonomous and normal car drivers.

To find the optimal share, we solve the first-order conditions of Eq.(11) ${ }^{3}$. Comparing the outcomes with those under marginal cost provision in Table 5, we can obtain Proposition 1. The proof is given in Appendix B.

Proposition 1 Consider situations when second-best public and private provision lead to the same case, i.e. both have "High" or "Low" proportion of autonomous car users, then for interior solutions,

\footnotetext{
${ }^{3}$ The second-order derivative satisfies $\frac{d^{2} S W}{d f^{2}}=\frac{d M S B}{d f}<0$, hence the maximization can be reached.
} 
(i) for Case $1 \mathrm{H}$ and $1 \mathrm{~L}, f^{p u b}=\frac{1}{2} f^{M C}+\frac{\gamma}{2\left(\beta^{\prime}+\gamma\right)}$;

(ii) for Case $2 \mathrm{H}$ and $2 \mathrm{~L}, f^{p u b}=\frac{1}{2} f^{M C}+\frac{\beta}{2\left(\beta+\gamma^{\prime}\right)}$;

(iii) for Case $3, f^{p u b}=\frac{1}{2} f^{M C}$.

Proposition 1 indicates that the travel pattern matters in determining the provision strategy. Indeed, the relationship between the equilibrium shares of second-best public versus marginal cost provision depends on the marginal external benefit to all drivers, which are related to the equilibrium travel patterns. When the negative marginal external benefit to the autonomous car drivers dominates the positive one to the normal car drivers, i.e. $M E B_{a}+M E B_{n}<0$, marginal cost provision leads to an over-supply of the autonomous cars. Therefore, $f^{P u b}$ is below $f^{M C}$ and a tax on autonomous cars is required to correct the negative externality. Otherwise, $f^{P u b}$ is above $f^{M C}$ and a subsidy is required.

\subsection{Private monopoly provision}

The private monopolist maximizes its profit, which equals the mark-up per trip multiplied by the number of autonomous cars. The profit maximization problem is:

$$
\max _{f, M U_{a}} \Pi=\max _{f, M U_{a}} M U_{a} \cdot f N=\max _{f, M U_{a}}\left\{\begin{array}{l}
\left(\Delta u_{H}(f)-M C_{a}\right) \cdot f N, \quad \text { if } F^{\#} \leq f \leq 1, \\
\left(\Delta u_{L}(f)-M C_{a}\right) \cdot f N, \quad \text { if } 0 \leq f \leq F^{\#}
\end{array}\right.
$$

where $\Pi$ is the monopolist's profit. $\Delta u_{H}$ and $\Delta u_{L}$ give the willingness to pay for the autonomous car in the high-share and low-share cases, respectively.

As there are two cases, there are two local maximal in which the first- and second-order conditions for Eq.(13) hold. The monopolist chooses the one that maximizes its profit; or, as we will see, may also choose the corner solution, exactly between the two cases. At this point separating the low and high case, the first order condition is discontinuous and has a massive jump. For now in the analytial model, we will focus more on the interior solutions. We will consider the corner solution in the numerical model.

For interior solutions, solving the first-order condition of Eq.(13) yields the mark-up on the autonomous cars under monopoly pricing:

$$
M U_{a}=-M E B_{a}-M E B_{n}-\frac{\partial c_{n}}{\partial f}
$$

where the last term measures the effect of autonomous cars on the normal car drivers' travel cost, which is positive. This implies that compared to the second-best mark-up, the private monopolist 
internalizes the negative external effects that autonomous cars imposed on the autonomous car drivers themselves, but ignores the positive ones on normal cars. The profit-maximizing pricing hence exceeds the second-best welfare-maximizing pricing.

The mark-up on the autonomous car in Eq.(14) can also be rewritten as

$$
\begin{aligned}
M U_{a} & =\left(\frac{\partial c_{a}}{\partial N_{a}}-\frac{\partial c_{n}}{\partial N_{a}}\right) N_{a}-\left(\frac{\partial c_{a}}{\partial N_{n}}-\frac{\partial c_{n}}{\partial N_{n}}\right) N_{a} \\
& =M E C_{a}-M E C_{n}-\left(\frac{\partial c_{n}}{\partial N_{a}}-\frac{\partial c_{n}}{\partial N_{n}}\right) N
\end{aligned}
$$

where the first term in Eq.(15) captures the effect of autonomous car on the willingness to pay, and the second term captures the effect of normal car on the willingness to pay. The $M E C_{i}$ is defined as the "full" marginal external cost, imposed by type $i$ cars on both types of users. The last term in Eq.(16) gives the monopolist mark-up due to the market power.

The optimal share can also be obtained by solving the first-order condition of Eq.(13). For interior solutions, we find that the optimal share under profit maximizing pricing with a private monopoly is half of that under marginal cost pricing, regardless of the travel patterns. This conclusion is presented in Proposition 2.

Proposition 2 Consider interior solutions, the optimal share for a private monopolist is half of that under marginal cost pricing, i.e. $f^{P M}=\frac{1}{2} f^{M C}$.

Recalling that when the travel pattern is in Case 3, the second-best optimal share is also half of that under marginal cost pricing (see Proposition 1), we can easily conclude that private monopoly provision equals the second-best socially optimum provision at this time. We will discuss it in more detail in Section 5.4.

\section{Price-sensitive demand}

A first important extension is to make demand price-sensitive. Thus, if autonomous cars make travel cheaper, one might expect to see more car users, and this is important to consider. We are again interested in the pricing rule and relative efficiency in perfect competition and with a profit-maximizing monopoly, compared to the second-best social optimum.

We assume that drivers regard the two alternatives as perfect substitutes. There is therefore one aggregate inverse demand function $D(N)$, where $N$ denotes the total number of travelers. The markup and the marginal cost of normal cars are again normalized to zero. The decision variables are the 
mark-up on the autonomous car $M U_{a}$, the number of autonomous and normal cars $N_{a}$, and $N_{n}$. In user equilibrium, the inverse demand equals the generalized utility of each mode:

$$
D(N)=-u_{n}\left(N_{a}, N_{n}\right), \quad D(N)=-u_{a}\left(N_{a}, N_{n}\right)+M C_{a}+M U_{a},
$$

where $u_{n}$ and $u_{a}$ are the utility of normal cars and autonomous cars. Note that each $\theta_{H} / \theta_{W}$ again applies for a high and low share of autonomous car owners. If $N_{a} /\left(N_{a}+N_{n}\right) \leq F^{\#}$, the utilities $u_{a}$ and $u_{n}$ correspond to the low-share case, otherwise, they correspond to the high-share case.

\subsection{Marginal cost provision}

With marginal cost provision, under perfect competition, the price of an autonomous car will equal its marginal cost, implying a zero mark-up. The interior equilibrium solutions of $N_{a}$ and $N_{n}$ are therefore determined by the following equation set,

$$
\left\{\begin{array}{l}
M U_{a}=0, \\
D(N)=-u_{n}\left(N_{a}, N_{n}\right), \\
D(N)=-u_{a}\left(N_{a}, N_{n}\right)+M C_{a} .
\end{array}\right.
$$

Given a specific $D(N), N_{a}$ and $N_{n}$ can be endogenously determined. For corner solution of $N_{n}=0$, we have $D\left(N_{a}\right)=-\left(u_{a}\left(N_{a}, 0\right)-M C_{a}\right)$, and that of $N_{a}=0$, we have $D\left(N_{n}\right)=-u_{a}\left(0, N_{n}\right)$.

\subsection{Second-best public provision}

A social planner aims to maximize social welfare by setting the mark-up of autonomous cars and the number of autonomous and normal cars. Social welfare is defined as the total consumer benefit,

minus the congestion costs and the automobile cost. When both drivers travel on the road, i.e. $N_{a}>0$, $N_{n}>0$, the Lagrangian is given by:

$$
\begin{aligned}
\max _{N_{a}, N_{n}, M U_{a}} S W= & \int_{0}^{N_{a}+N_{n}} D(x) d x+u_{a}\left(N_{a}, N_{n}\right) \cdot N_{a}+u_{n}\left(N_{a}, N_{n}\right) \cdot N_{n}-M C_{a} \cdot N_{a} \\
& -\lambda_{a}\left(D(N)+u_{a}\left(N_{a}, N_{n}\right)-M C_{a}-M U_{a}\right)-\lambda_{n}\left(D(N)+u_{n}\left(N_{a}, N_{n}\right)\right),
\end{aligned}
$$

where the Lagrangian multipliers $\lambda_{a}$ and $\lambda_{n}$ refer to the user equilibrium constraints.

For interior solutions, we have

$$
\begin{aligned}
M U_{a} & =-\frac{\partial u_{a}}{\partial N_{a}} N_{a}-\frac{\partial u_{n}}{\partial N_{a}} N_{n}-\left(\frac{-D^{\prime}-\frac{\partial u_{n}}{\partial N_{a}}}{-D^{\prime}-\frac{\partial u_{n}}{\partial N_{n}}}\right)\left(-\frac{\partial u_{a}}{\partial N_{n}} N_{a}-\frac{\partial u_{n}}{\partial N_{n}} N_{n}\right) \\
& =M E C_{a}-\frac{\Delta N_{n}}{\Delta N_{a}} M E C_{n} .
\end{aligned}
$$


The derivation is in Appendix D.

The first two terms in Eq.(20) are the marginal external costs imposed by autonomous cars in the second-best equilibrium. The last term corrects for the marginal costs of congestion caused by substitution to the normal cars when a mark-up is levied on autonomous cars. The marginal external costs imposed by normal cars are multiplied by a fraction which depends on the sensitivity of the marginal benefits with respect to total demand, both systematically via $D^{\prime}(N)$ and via the sensitivity of the utility of normal cars with respect to the number of the autonomous cars $\frac{\partial u_{n}}{\partial N_{a}}$ and to the number of normal cars $\frac{\partial u_{n}}{\partial N_{n}}$.

A more detailed look at Eq.(20) shows that the $M U_{a}$ can be rewritten as $M U_{a}=M E C_{a}-$ $\frac{\Delta N_{n}}{\Delta N_{a}} M E C_{n}$, as expressed in Eq.(21), where $M E C_{i}$ is the marginal external cost imposed by type $i$ cars on both car drivers. It means that switching to autonomous car increases the welfare loss from normal cars. More specifically, Eq.(21) shows that the term $-D^{\prime}-\frac{\partial u_{n}}{\partial N_{a}}$ is the change in the constraint for normal cars due to a marginal change in $N_{a}$, whereas $-D^{\prime}-\frac{\partial u_{n}}{\partial N_{n}}$ is the change in the constraint for normal cars due to a marginal change in $N_{n}$. The ratio therefore gives $\frac{\Delta N_{n}}{\Delta N_{a}}$.

For perfectly overall inelastic demand, $D^{\prime}(N) \rightarrow-\infty$, the $M U_{a}$ rule reduces to the difference in marginal external costs of both users:

$$
\lim _{D^{\prime}(N) \rightarrow-\infty} M U_{a}=\left(-\frac{\partial u_{a}}{\partial N_{a}} N_{a}-\frac{\partial u_{n}}{\partial N_{a}} N_{n}\right)-\left(-\frac{\partial u_{a}}{\partial N_{n}} N_{a}-\frac{\partial u_{n}}{\partial N_{n}} N_{n}\right)
$$

This is consistent with Eq.(12). Because there is no effect of $M U_{a}$ on the overall demand, the regulator only seeks to find the optimal mode split.

In contrast to the zero mark-up under marginal cost pricing, a positive second-best mark-up means that marginal cost provision leads to an over-supply of autonomous cars, and a corrective tax is needed. A negative second-best mark-up means that marginal cost provision leads to an under-supply of autonomous cars and a subsidy is needed. If the second-best mark-up is also zero, it implies that marginal cost provision equals the second-best optimum provision.

\subsection{Private monopoly provision}

A private monopolist would maximize profits by solving the following Lagrange problem:

$$
\begin{aligned}
\max _{N_{a}, N_{n}, M U_{a}} \Pi= & M U_{a} \cdot N_{a} \\
& -\lambda_{a}\left(D(N)+u_{a}\left(N_{a}, N_{n}\right)-M C_{a}-M U_{a}\right)-\lambda_{n}\left(D(N)+u_{n}\left(N_{a}, N_{n}\right)\right) .
\end{aligned}
$$


For interior solutions, we find

$$
\begin{aligned}
M U_{a} & =\left(-\frac{\partial u_{a}}{\partial N_{a}}+\frac{\partial u_{n}}{\partial N_{a}}\right) N_{a}-\left(\frac{-D^{\prime}-\frac{\partial u_{n}}{\partial N_{a}}}{-D^{\prime}-\frac{\partial u_{n}}{\partial N_{n}}}\right)\left(-\frac{\partial u_{a}}{\partial N_{n}}+\frac{\partial u_{n}}{\partial N_{n}}\right) N_{a} \\
& =\left(-\frac{\partial u_{a}}{\partial N_{a}}+\frac{\partial u_{n}}{\partial N_{a}}\right) N_{a}-\frac{\Delta N_{n}}{\Delta N_{a}}\left(-\frac{\partial u_{a}}{\partial N_{n}}+\frac{\partial u_{n}}{\partial N_{n}}\right) N_{a} .
\end{aligned}
$$

The first term of Eq.(24) is the difference of marginal costs on autonomous cars and normal cars imposed by autonomous cars. The last term corrects for the marginal cost difference caused by substitution to the normal cars when a $M U_{a}$ is levied on autonomous cars. The marginal cost difference imposed by the normal cars is multiplied by a fraction, which depends on the sensitivity of the marginal benefits with respect to the demand, $\frac{\partial u_{n}}{\partial N_{a}}, \frac{\partial u_{n}}{\partial N_{n}}$ and of the demand curve $D^{\prime}(N)$. Using the same logic

as in second-best public provision, we find the ratio again gives $\frac{\Delta N_{n}}{\Delta N_{a}}$, as expressed in Eq.(25). In contrast to the second-best public provision, where the correction can be positive or negative, for the monopoly provision, a non-positive mark-up has to be subtracted. Clearly, the monopolistic mark-up will never be a subsidy.

Compared to the second-best mark-up in Eq.(21), the mark-up with a monopolist can be further written as

$$
M U_{a}=M E C_{a}-\frac{\Delta N_{n}}{\Delta N_{a}} M E C_{n}-\left(\frac{\partial c_{n}}{\partial N_{a}}-\frac{\Delta N_{n}}{\Delta N_{a}} \frac{\partial c_{n}}{\partial N_{n}}\right) N
$$

implying that the profit-maximizing mark-up exceeds the second-best welfare-maximizing mark-up. This is because the private monopolist internalizes the negative external effects that the autonomous cars impose on autonomous car drivers themselves. However, the positive externalities of autonomous cars to the normal car drivers are (as with inelastic demand) left outside the monopolistic pricing rule, and this term would reduce the socially optimal price. In addition, with price-sensitive demand, the markup is also demand-related. The effect of the price elasticity depends on the relative strength of the marginal external cost of autonomous cars and the correction on the marginal cost of normal cars (the first two terms in Eq.(26)), and the monopolistic mark-up determined by the market power, the last term in Eq.(26).

Apart from the interior solutions, the corner solution is often common. When the share of autonomous car drivers is at the critical point, mark-up is determined by the Wardrop equilibrium with a fixed share of autonomous cars $F^{\#}$.

\subsection{A detailed discussion on Case 3}

Case 3 is a special case that may switch to Case $1 \mathrm{H}$ or Case $2 \mathrm{H}$ as the share of autonomous car increases, so we analyze it more specifically in this subsection. The following proposition reveals the 
properties of the mark-up under second-best public and private monopoly provision in Case 3 .

Proposition 3 If the travel pattern is in case 3, (i) private monopoly provision equals the second-best socially optimum provision; and (ii) for second-best public and private monopoly provision, $\frac{\partial M U_{a}}{\partial D^{\prime}}=0$, $\frac{\partial M U_{a}}{\partial \theta_{H}}>0, \frac{\partial M U_{a}}{\partial \theta_{W}}=0$.

To understand Proposition 3, we first look at the fixed demand case. The travel cost of normal

car drivers is constant at $\frac{\delta N}{s}+\alpha T_{f}$, so the marginal external benefit enjoyed by normal car drivers is zero. The mark-up for the second-best public and private monopoly supplier thus both reduce to $-M E B_{a}$. This also means that no demand-related price premium can be applied: the effective demand for autonomous cars is perfectly elastic because the user cost for normal cars is constant. Moreover, the optimal share provided in these two regimes are both half of that under marginal cost provision (see Proposition 1 and Proposition 2). Private monopolistic provision hence also maximizes social welfare.

Now we need to prove that price sensitivity does not change the total number of cars. Apparently, the total number of cars satisfies the user equilibrium condition:

$$
D(N)=\delta N / s+2 \alpha T_{f} .
$$

Note that the right-hand side of Eq.(27) is also the utility one would obtain if all users have normal cars. The total number of cars is hence also always at $N$ regardless of the price elasticity, since Eq.(27) will remain true for marginal changes of the monopoly price. The price-sensitive demand then reduces to the fixed demand case.

In addition, substituting $f^{p u b}=f^{P M}=1 / 2 f^{M C}$ into Eq.(12) yields

$$
M U_{a}=\frac{\theta_{H} \delta N-\alpha\left(M C_{a}-2 \theta_{H} T_{f}\right) s}{2 \alpha N},
$$

where $f^{M C}$ is given in Table 4 .

Taking the derivative of Eq.(28) yields

$$
\frac{\partial M U_{a}}{\partial D^{\prime}}=0, \frac{\partial M U_{a}}{\partial \theta_{H}}=\frac{\delta+2 \alpha s T_{f}}{2 \alpha}>0, \frac{\partial M U_{a}}{\partial \theta_{W}}=0 .
$$

\section{$6 \quad$ Numerical example}

This section presents numerical results for two base calibrations of the model developed above: one for the fixed demand and one for the price-sensitive demand, both under marginal cost pricing, second-best public pricing and profit maximizing pricing with a private monopolist. We also analyze the performance of these three provision regimes. After discussing these base cases, we turn to the 
sensitivity analyses. The model outcomes are rather sensitive to the parameters, underlining the importance to present these results.

\subsection{Calibration of the numerical models}

For the calibration, we stay close to Van den Berg and Verhoef (2016), and focus on petrol passenger cars. The schedule delay parameters will be based on the ratios $\beta / \alpha=39 / 64$ and $\gamma / \alpha=1521 / 640$ established in Small (1982), as are common in the literature. The total number of users under inelastic demand is 9,000 , and the capacity of the bottleneck is 3600 . We consider a trip of $20 \mathrm{~km}$, with a free-flow travel time of 20 minutes, implying a free flow speed of $120 \mathrm{~km} / \mathrm{hr}$. We use a VOT $(\alpha)$ of $€ 10$, and an $M C_{a}$ of $€ 1.51$ (Van den Berg and Verhoef (2016)).

For the price sensitive demand, it is assumed that a linear inverse demand function applies:

$$
D(N)=a-d N
$$

A choice of $a=72.48$ and $d=0.006$ ensures that the price elasticity of demand is -0.35 in the base equilibrium, and when there are no autonomous cars, the number of normal car drivers is 9000 . We assume that the possible substitution for home is $€ 4$ and for work is $€ 1$, i.e. $\theta_{H}=4, \theta_{W}=1$, implying that autonomous driving is a closer substitute to being at home than to being at work. Therefore, the

possible travel pattern is Case $1 \mathrm{H}$ or Case $1 \mathrm{~L}$, and the critical share to distinguish these two cases is 0.82 .

\subsection{Base case}

Table 4 shows the outcomes of the base calibrations for four cases: no autonomous vehicles (No AV), marginal cost provision (MC), second-best public provision (Pub), and private monopoly provision $(\mathrm{PM})$. Relative efficiency is the total social welfare improvement of a policy from the case without autonomous cars, divided by the welfare improvement under socially optimal public provision.

Consistent with expectations and with the result in Van den Berg and Verhoef (2016), the introduction of autonomous cars lowers costs, and raises utility. Of course, individual users can never be worse off than before from adopting, as they can always all choose to stick to the normal car. We find that impacts on congestion externalities do not undermine this intuition. As expected, the mark-up under private monopoly provision exceeds that under second-best public provision.

In terms of the equilibrium share of autonomous cars and the travel pattern on the road, different regimes of course show different results. Marginal cost pricing leads to $100 \%$ autonomous cars, because the autonomous car gives a higher utility than the normal car. Under second-best public provision, 


\section{Table 4}

Outcomes under the base calibrations.

\begin{tabular}{|c|c|c|c|c|c|c|c|c|}
\hline & \multicolumn{4}{|c|}{ Fixed demand } & \multicolumn{4}{|c|}{ Price-sensitive demand } \\
\hline & No AV & $\mathrm{MC}$ & Pub & $\mathrm{PM}$ & No AV & $\mathrm{MC}$ & Pub & $\mathrm{PM}$ \\
\hline$N$ & 9,000 & 9,000 & 9,000 & 9,000 & 9,000 & 9,891 & 9,567 & 9,215 \\
\hline$N_{a}$ & 0 & 9,000 & 8,550 & 7,380 & 0 & 9,891 & 8,802 & 7,556 \\
\hline$N_{n}$ & 9,000 & 0 & 450 & 1,620 & 9,000 & 0 & 765 & 1,659 \\
\hline$f$ & - & 1 & 0.95 & 0.82 & - & 1 & 0.92 & 0.82 \\
\hline$M U_{a}$ & - & 0 & 1.81 & 5.39 & - & 0 & 2.82 & 5.49 \\
\hline$S W$ & 0 & 57,142 & 57,800 & 53,700 & 240,190 & 293,536 & 297,710 & 294,880 \\
\hline$u_{n}$ & -18.79 & -12.82 & -14.09 & -17.26 & -18.79 & -13.43 & -15.41 & -17.51 \\
\hline$u_{a}$ & - & -10.93 & -10.77 & -10.36 & - & -11.62 & -11.08 & -10.51 \\
\hline$\omega^{*}$ & 0 & 0.99 & 1 & 0.93 & 0 & 0.93 & 1 & 0.95 \\
\hline
\end{tabular}

Note: ${ }^{*}$ Relative efficiency $\omega$ is the welfare gain of a policy relative to the case without autonomous cars divided by the gain from second-best public provision.

the optimal share is 0.95 . Now the travel pattern is Case $1 \mathrm{H}$, where the autonomous car drivers can be early or late for work, and the normal car drivers who arrive after the autonomous cars are late for work. For the private monopoly provision, the discontinuity makes the corner solution a more likely outcome than others. This implies that the private monopolist will choose the critical share, $82 \%$ of the autonomous cars. Compared to Case 1H, now normal car drivers still arrive after the autonomous cars, but all autonomous car drivers are early for work and all normal car drivers are late.

Under second-best public and private monopoly provision, allowing for price-sensitive demand raises the mark-up. Intuitively, when the demand becomes price-sensitive, the number of autonomous cars and normal cars both increase when the generalized price of traveling falls after introducing the autonomous car. This results in heavier congestion, which tends to raise the marginal external costs of both types of car user. For second-best public provision, the mark-up equals the marginal external costs of autonomous cars, minus the correction on the marginal external costs of normal cars. As the marginal change of the number of autonomous cars exceeds that of the normal cars, the increase in the marginal external cost of autonomous cars dominates the correction of normal cars. Hence the mark-up with price-sensitive demand is higher than the one applying with the fixed demand case. For private monopoly provision, considering the monopolist neglects the positive externality on normal car drivers, the mark-up thus also becomes higher than in the fixed demand case.

Although marginal cost provision provides $100 \%$ of autonomous cars, social welfare is still lower 
than that under second-best public provision. This reflects that a taxation is required to achieve higher social welfare, which would mean bringing down the number of autonomous cars from 9, 000 to 8,550 with a tax of 1.81 under fixed demand, and from 9,891 to 8,801 with a tax of 2.82 under price-sensitive demand. In addition, due to the monopolistic market power and the implied price premium, private monopoly even performs better than perfect competition when the demand is price-sensitive.

\subsection{Sensitivity analysis with respect to $\theta_{H}$ and $\theta_{W}$}

There is little to no guidance from the literature on what would be realistic values of $\theta_{H}, \theta_{W}, M C_{a}$, and the demand parameters. Hence, it is vital to do extensive sensitivity analyses. The effects of other parameters were found to be in line with the theoretical discussion, so these will not be discussed. Section 6.4 will look at variation in the demand parameters. Section 6.5 considers the marginal cost of autonomous cars $M C_{a}$. First, this section considers variation in $\theta_{H}$ and $\theta_{W}$ from 0.5 to 6.5 .

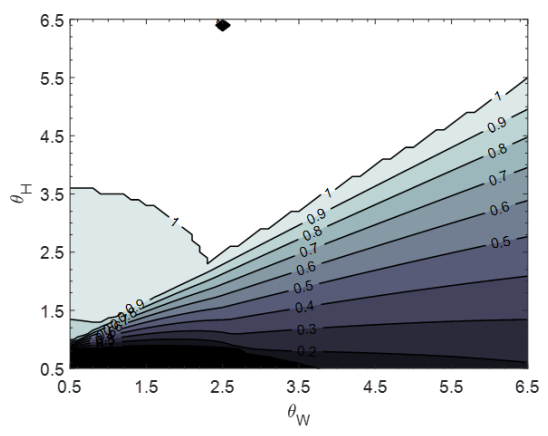

(a) Marginal cost pricing

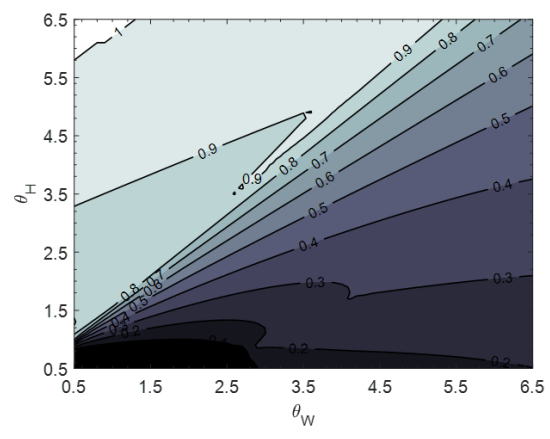

(b) Second-best public provision

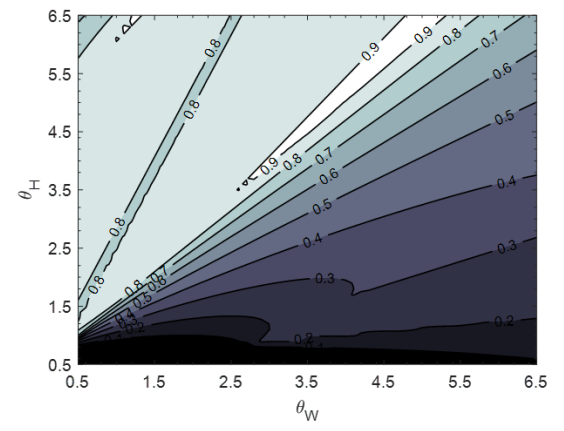

(c) Private monopoly

Fig. 4. Effect of $\theta_{H}$ and $\theta_{W}$ on equilibrium share of autonomous cars.

Fig.4 shows the equilibrium share of autonomous car with marginal cost pricing (a), second-best public pricing (b) and private monopoly provision (c). The darker the area of a contour plot is, the lower the share of the autonomous car. The kinks are induced by changes in the travel pattern. For a given $\theta_{W}$, the optimal share increases with $\theta_{H}$, but for a given $\theta_{H}$, it roughly decreases with $\theta_{W}$. Intuitively, the higher $\theta_{W}$ is, the more likely the autonomous car drivers are to travel in the peak. This raises the negative externality that autonomous cars impose on each other, which makes decreasing the share more beneficial. Conversely, a higher $\theta_{H}$ means the autonomous car drivers are more likely to travel in the shoulder of the bottleneck. This raises the positive externality on normal car drivers, which makes increasing the share more beneficial.

The marginal cost pricing may lead to second-best or over second-best optimal share due to the 
zero mark-up, while the private monopoly provision may lead to second-best share or to a lower share. For the corner solution of 1, the area of $100 \%$ autonomous cars under marginal cost provision is much larger than that under second-best public provision. More specifically, only when $\theta_{H}$ is sufficiently high and $\theta_{W}$ is sufficiently low, can the second-best public provide $100 \%$ autonomous cars. Whereas under marginal cost provision, it only requires $\theta_{H}>\theta_{W}$ and a sufficient larger $\theta_{H}$. Intuitively, a large $\theta_{H}$ and a small $\theta_{W}$ both make raising the share more beneficial, as the former lowers the travel cost and raises the willingness to pay for the autonomous car, and the latter also means a large $\theta_{H} / \theta_{W}$. Still, private monopoly provision will not lead to $100 \%$ autonomous cars due to the monopolistic market power. When $\theta_{H}$ and $\theta_{W}$ are both sufficiently low, no suppliers provides autonomous cars and all provision regimes lead to corner solution 0 .

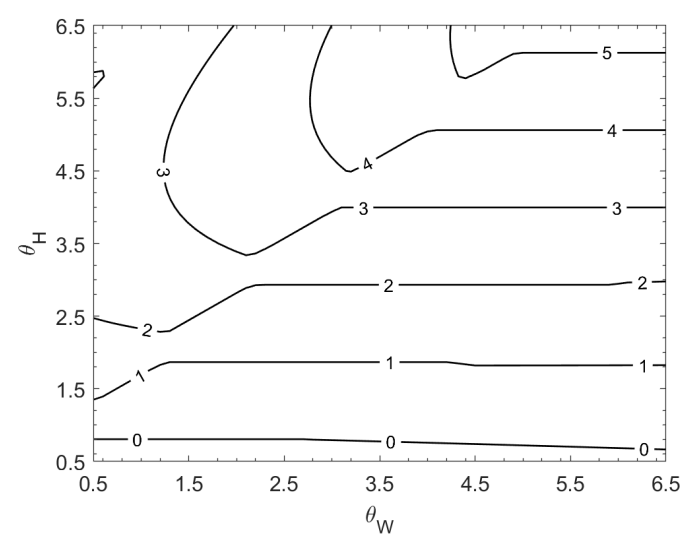

(a) Second-best public provision

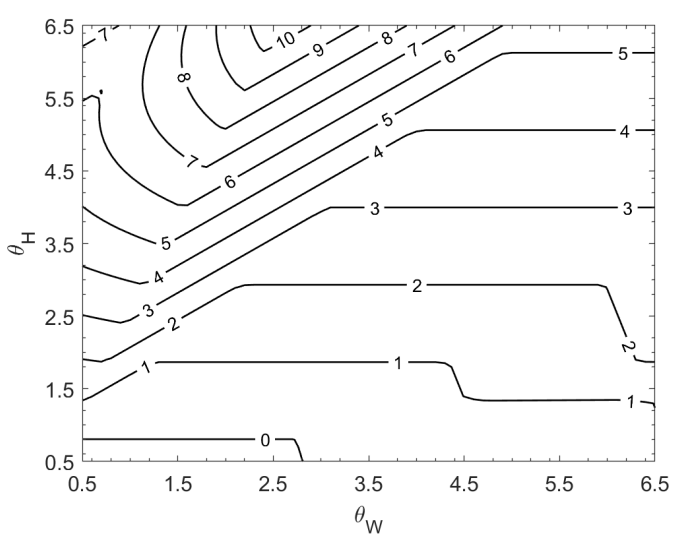

(b) Private monopoly

Fig. 5. Effect of $\theta_{H}$ and $\theta_{W}$ on the mark-up.

Fig.5 compares the changes in $M U_{a}$ under second-best public and private monopoly provision. Not surprisingly, the latter always equals or exceeds the former one, because the private provider like the public provider internalizes the negative external benefits that the autonomous car drivers impose up on one another, but adds a monopolistic mark-up. Moreover, the public operator lowers the mark-up to allow for more autonomous car drivers imposing a positive externality on normal car drivers. In the middle of the figure, there is a region where $M U_{a}$ does not change with $\theta_{W}$, and the mark-up provided by the monopolist is just the same as that by the second-best public provision. Indeed, this is consistent with our discussion in Section 5.4, as the travel pattern here is in Case 3. In addition, for both regimes, $\theta_{H}$ has a stronger effect than $\theta_{W}$, because $\theta_{H}$ does not only affect the value of time but also the schedule delay, while $\theta_{W}$ only affects the value of schedule delay.

Fig.6 shows the effects of $\theta_{H}$ and $\theta_{W}$ on the relative efficiency $\omega$, under (a) marginal cost provision, 


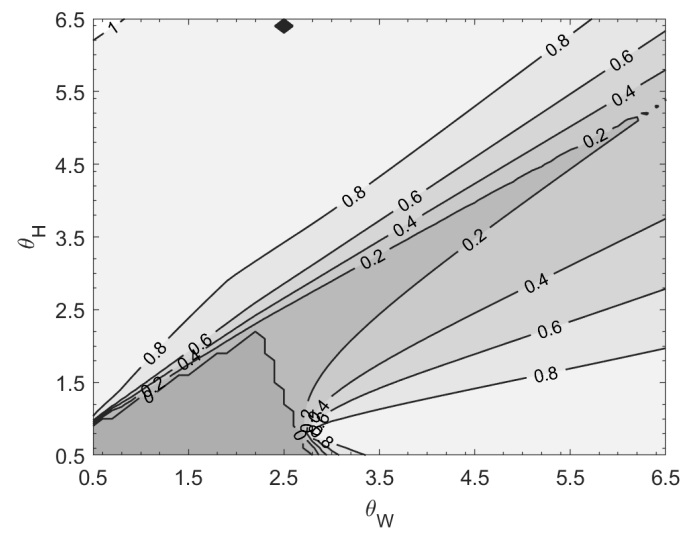

(a) Marginal cost pricing

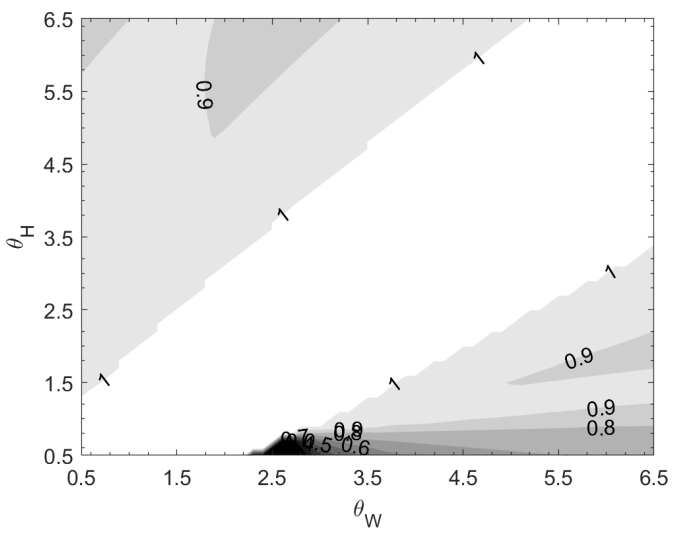

(b) Private monopoly

Fig. 6. Effect of $\theta_{H}$ and $\theta_{W}$ on the relative efficiency.

and (b) private monopoly provision. The darker the area, the lower the relative efficiency. In the white area of Fig.6(b), the private monopoly provision is also second-best. However, for the same region in Fig.6(a), the relative efficiency varies between 0 to 0.8 , as the zero mark-up is lower than the second-best mark-up. The zero mark-up thus brings over-consumption and makes perfect competition harmful. For the private monopoly provision, the travel pattern in this region is Case 3. Hence, the relative efficiency of 1 follows directly from the discussion in Section 5.4.

\subsection{Sensitivity analysis with respect to demand parameters}

Fig.7 shows the effects of demand parameters on the mark-up. As different pairs of $\left(\theta_{H}, \theta_{W}\right)$ may result in different travel patterns on the road, we show results for $\theta_{H}=1, \theta_{W}=1.5$ in Fig.7(a); $\theta_{H}=2.5, \theta_{W}=1.5$ in Fig.7(b); $\theta_{H}=4.5, \theta_{W}=1.5$ in Fig.7(c); and finally $\theta_{H}=6.5, \theta_{W}=1.5$ in Fig.7(d). For interior solutions, the mark-up under second-best public provision equals the difference of the marginal external costs of autonomous cars compared to normal cars, corrected for the fact that this mark-up also distorts the overall demand. Under private monopoly provision, monopoly power also plays an important role. In addition, as we have noted before, the supplier will also choose the corner solution that exactly separates the high and low share case, so that the travel pattern is just at the critical case that between the two cases.

Fig.7(a-b) show that when the travel pattern is in Case 3, second-best public and private monopoly provision lead to the same mark-up, and the mark-up does not change with the slope of the inverse demand. This result is consistent with our discussion in Proposition 3, which implies that in case 3, private supply does not lead to market power distortions. 


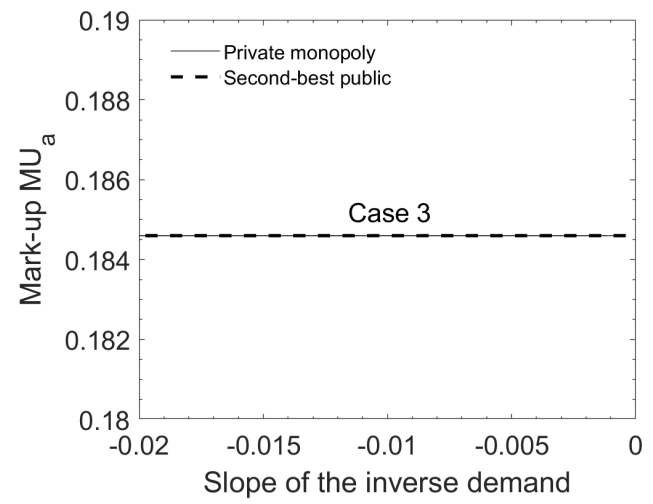

(a) $\theta_{H}=1, \theta_{W}=1.5$

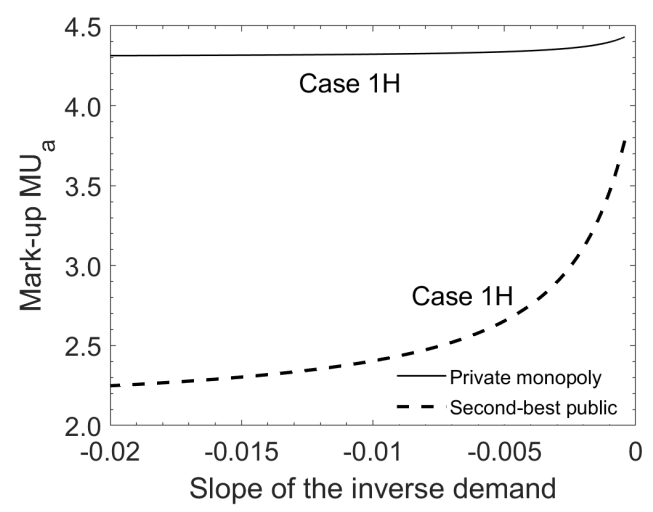

(c) $\theta_{H}=4.5, \theta_{W}=1.5$

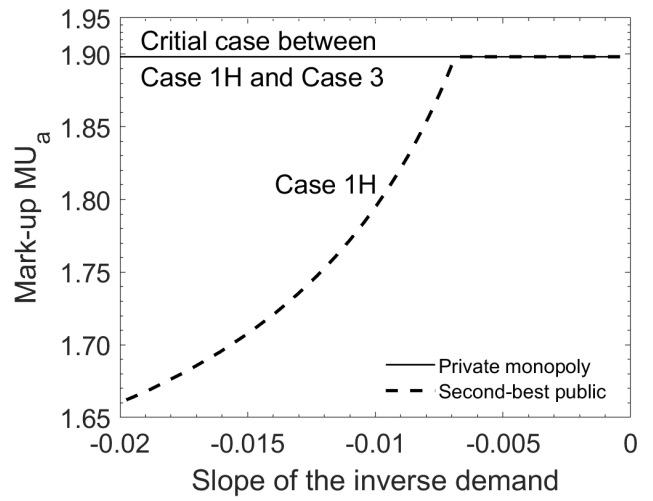

(b) $\theta_{H}=2.5, \theta_{W}=1.5$

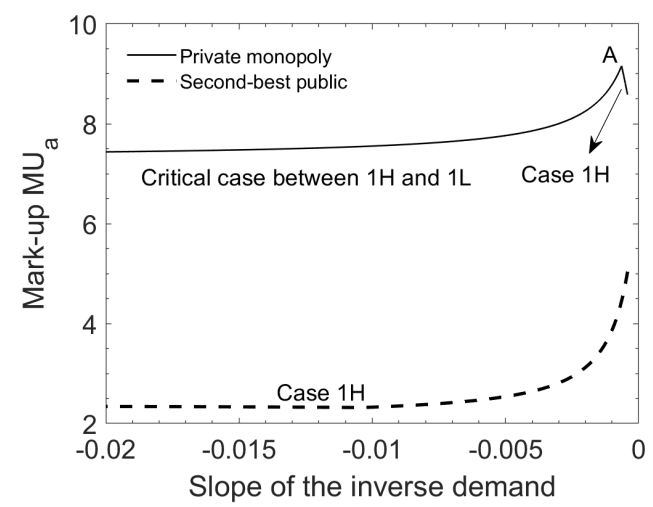

(d) $\theta_{H}=6.5, \theta_{W}=1.5$

Fig. 7. Effect of demand parameters on $M U_{a}$.

Fig.7(c) shows that for second-best public and private monopoly provision, the travel patterns are both in Case 1H. Surprisingly, as demand becomes more price-sensitive towards the right-hand side of the diagrams, the mark-ups both increase, whereas the difference between the mark-ups in the two regimes decreases. Indeed, total demand increases with the price elasticity, leading to more cars and congestion on the road. So the more price-sensitive the demand is, the higher the marginal external costs of both cars. This raises the second-best mark-up. For the private monopoly provision, the monopolistic mark-up is decreasing, but the monopolistic market power is not strong enough to overpass the increasing of the marginal external costs. Consequently, the mark-up under private monopoly also increases with the slope of the inverse demand, but less so than the second-best public.

Fig.7(d) shows that when $\theta_{H}=6.5, \theta_{W}=1.5$, the mark-up of the private monopoly first increases but then starts to fall when the inverse demand becomes increasingly flat. Indeed, before point $A$, the number of autonomous and normal cars both increase, and the mark-up increases with the price 
elasticity due to the increasing congestion effects. From point A on, the number of normal cars starts to fall, and that of autonomous cars still increases and more rapidly than before. This leads to increasing marginal external costs. However, the positive externality on the normal cars is decreasing. The declining monopolistic market power thus tends to reduce the monopolistic mark-up, and this effect is even stronger than the congestion effects. Consequently, the mark-up starts to fall after point $A$.

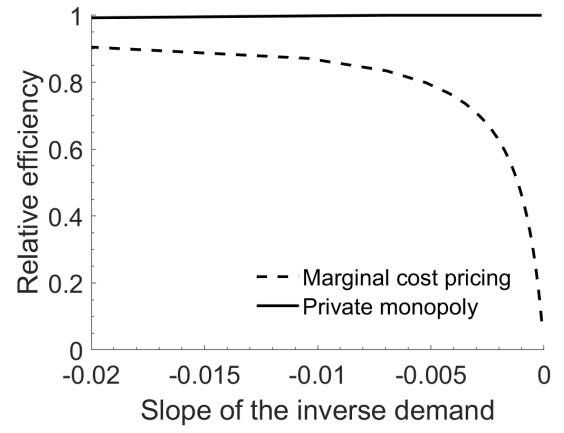

(a) $\theta_{H}=2.5, \theta_{W}=1.5$

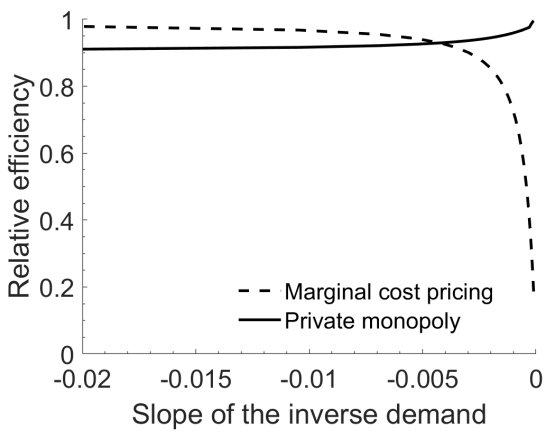

(b) $\theta_{H}=4.5, \theta_{W}=1.5$

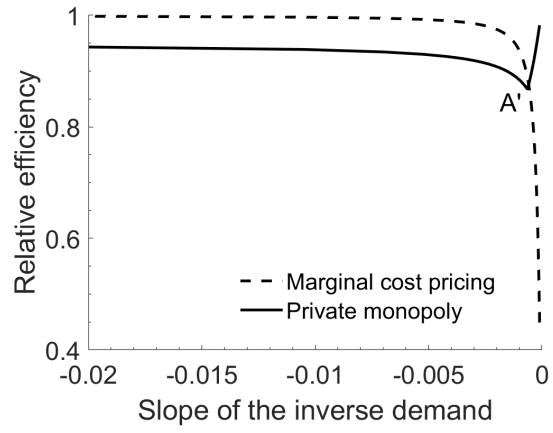

(c) $\theta_{H}=6.5, \theta_{W}=1.5$

Fig. 8. Effect of demand parameters on relative efficiency.

Fig. 8 further presents the relative efficiency of marginal cost pricing and the private monopoly. ${ }^{4}$ A higher price elasticity lowers the social welfare for all regimes. Under marginal cost provision, the relative efficiency drops with the slope of the inverse demand due to the congestion effect. This is consistent with the increasing $M U_{a}$ in Fig.7. Under private monopoly provision, the relative efficiency depends on the relative strength of the externality in second-best public provision, the externality in private monopoly provision, and the monopolistic market power effect. The first and third term tend to raise the relative efficiency, and the second term tends to reduce it. When $\theta_{H}=4.5, \theta_{W}=1.5$ in Fig.8(b), the externality in second-best public provision dominates, leading to an increasing relative efficiency. When $\theta_{H}=6.5, \theta_{W}=1.5$ in Fig.8(c), the externality in private monopoly dominates before $A^{\prime}$, and then the monopolistic market power dominates afterwards. So the relative efficiency first drops and then increases. This is consistent with Fig.7(c).

\subsection{Sensitivity analysis with respect to $M C_{a}$}

Fig.9 illustrates the effects of the marginal cost of autonomous cars $M C_{a}$ on the equilibrium share (a) and the mark-up of autonomous cars (b) with $\theta_{H}=4$ and $\theta_{W}=1$. The kinks are again induced

\footnotetext{
${ }^{4}$ When $\theta_{H}=1, \theta_{W}=1.5$, the relative efficiency of the marginal cost pricing is zero and the private monopoly provision is 1 . We do not present that result graphically.
} 


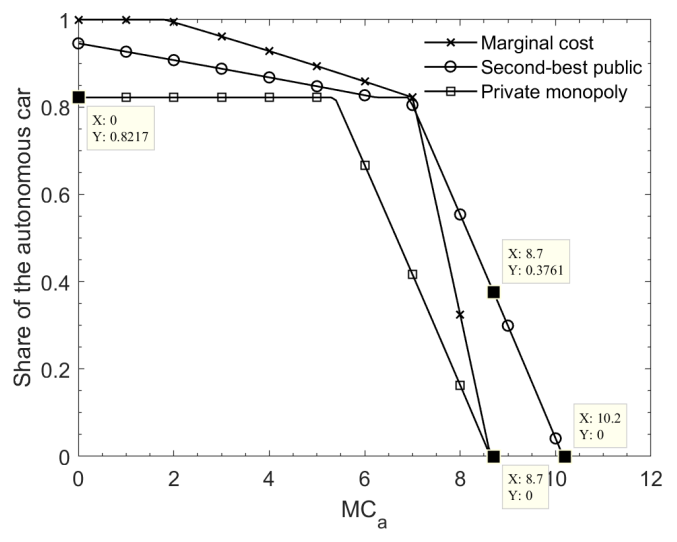

(a) Optimal share of the autonomous cars

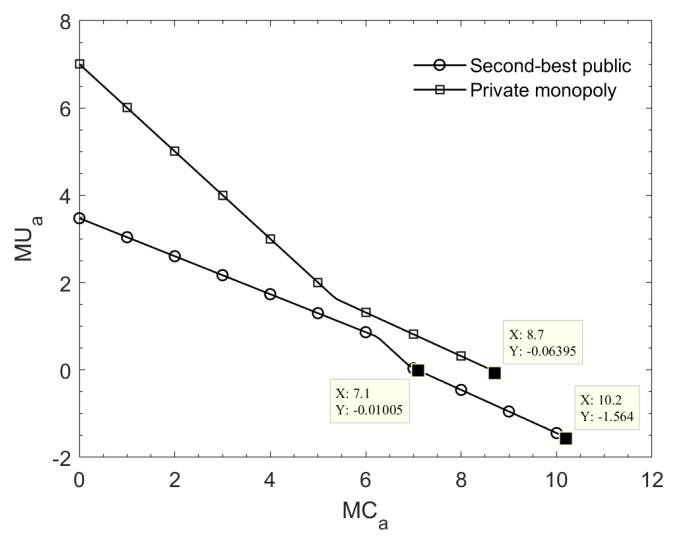

(b) Mark-up $M U_{a}$

Fig. 9. Effects of $M C_{a}$.

by a change of travel patterns. Fig.9(a) shows that when $M C_{a}$ is sufficient low, marginal cost pricing and private monopoly both lead to corner solutions. The difference is that in the former scheme, the equilibrium share is at $100 \%$, and in the latter the optimal share is always at the critical share 0.8217 . For interior solutions, the equilibrium share naturally decreases with $M C_{a}$. When $M C_{a}$ is over 8.7, both perfect competition and private monopolist no longer provide autonomous cars, due to the high cost, but second-best public provision still provides these as a measure to reduce congestion, until $M C_{a}$ reaches 10.2 .

Fig.9(b) further explains the reason, by showing the effects on $M U_{a}$. When $M C_{a}$ is lower than 7.1, the mark-up under second-best public provision is always positive, meaning that marginal cost pricing leads to over-supply of autonomous cars and the government taxes for them; when $M C_{a}$ exceeds 7.1, the mark-up becomes negative, meaning that the marginal cost provision leads to under-supply of the autonomous cars and the government subsidizes them. For private monopoly provision, the monopolist stops providing the autonomous cars once the mark-up becomes zero.

\section{Conclusion}

This paper considered the impacts of autonomous cars on dynamic bottleneck congestion patterns. We examined different types of market supply: marginal cost pricing, second-best public pricing, and profit-maximizing pricing by a private monopoly.

Our study shows some important findings and new insights. (i) Our results reveal that travel pattern changes are related to the changes of values of time and schedule delay that autonomous cars may bring 
about, as well as to the share of autonomous cars. In particular, if the time spent in the autonomous car is spent more easily on work activities than on leisure, autonomous car users will travel in the later part of the peak. Conversely, if these cars are more suitable for leisure, autonomous car users will arrive early. And finally and quite naturally, in between these cases, autonomous cars will arrive in the middle of the peak. (ii) As the autonomous car brings a positive externality on normal car drivers and a negative externality on autonomous car drivers, the net externality of an additional switcher from a normal car to an autonomous car can be positive or negative. The second-best public mark-up equals the difference of the marginal external costs of autonomous cars compared to normal cars, corrected for the fact that this mark-up also distorts the overall demand. For private monopoly provision, the market power effect also plays an important role. The mark-up of the monopolist is never lower than the second-best public one, but may be equal so that private supply would not lead to market power distortions. In particular, in the between case where autonomous cars use the center of the peak, the monopolist always sets the same price as the public supplier. So, the societal performance of provision regimes depends heavily on the effects of autonomous cars on the travel preferences. (iii) The difference of the mark-up between the second-best public and private monopoly provision becomes smaller as the slope of the inverse demand increases. Specifically, for public provision, the interior mark-up always increases with the slope of the inverse demand. But for private monopoly provision, it depends on the relative strength of the marginal external cost and the marker power effects. If the former is stronger, the mark-up increases; otherwise, it decreases.

Although this paper provides some new insights into the bottleneck model with autonomous cars, some important questions deserve attention in future research efforts. First, this paper mainly focused on " $\alpha-\beta-\gamma$ " preference, the properties of the model may not be applicable to the case with general scheduling preferences. In addition, empirical evidence on the shape of marginal utility functions is still limited. When the marginal utility functions are unknown, a trial-an-error iterative approach proposed by and may be applied. Second, we only consider one supplier, but in reality, there will be multiple suppliers of autonomous cars who may also provide normal cars. The competition and cooperation between these suppliers will be an interesting topic in the future research. Third, this study focused on a single road bottleneck, and thus the congestion effects over space cannot be addressed. To do so, a network traffic assignment model should be developed. In a further study, this paper can be extended to a network context such that the network congestion effects can be explicitly taken into account. 


\section{Acknowledgements}

Financial support from National Natural Science Foundation of China (71525003) and China Scholarship Council (201706160163) is gratefully acknowledged. Any remaining errors are ours.

\section{Appendix A The conditions for each pattern to happen.}

We illustrate with Case $1 \mathrm{H}$. We first consider the preference conditions. Substituting $\gamma^{\prime}=\gamma+\theta_{H}-$ $\theta_{W}$ and $\alpha^{\prime}=\alpha-\theta_{H}$ into $\gamma^{\prime} / \alpha^{\prime}>\gamma / \alpha$ yields $\theta_{H} / \theta_{W}>\alpha /(\alpha+\gamma)$.

Then we characterize the critical time boundaries for this case. Due to the heterogeneity induced by the in-vehicle activities, autonomous cars arrive in $\left[t_{1}, t_{3}\right]$, and normal cars arrive in $\left[t_{3}, t_{4}\right]$. The total commuting time should allow all commuters to pass the bottleneck, and the commuting time for autonomous cars should allow the whole autonomous car drivers to pass the bottleneck:

$$
t_{4}-t_{1}=N / s, \quad t_{3}-t_{1}=f N / s
$$

In equilibrium, the drivers of the same type car should have the same costs no matter when they arrive:

$$
-\beta^{\prime} t_{1}=\gamma^{\prime} t_{3}+\alpha^{\prime} T_{3}, \quad \gamma t_{4}=\alpha T_{3}+\gamma t_{3}
$$

where $T_{3}$ is the queuing time at $t=t_{3}$.

From the above equations, we can solve for $t_{1}, t_{3}$ and $t_{4}$.

$$
t_{1}=-\frac{\alpha^{\prime}(1-f) \gamma N+\alpha f \gamma^{\prime} N}{\alpha(\beta+\gamma) s} ; t_{3}=\frac{\alpha^{\prime}(1-f) \gamma N-\alpha \beta^{\prime} f N}{\alpha(\beta+\gamma) s} ; t_{4}=\frac{\alpha \beta^{\prime} N-\left(\alpha^{\prime} \gamma-\alpha \gamma^{\prime}\right)(1-f) N}{\alpha(\beta+\gamma) s} .
$$

To determine $t_{2}$, note that the first and last drivers of normal car drivers face no queue and should have the same cost at equilibrium if there is no autonomous car drivers:

$$
-\beta t_{2}=\gamma t_{4}
$$

By solving the time conditions in Table 1, i.e., $t_{3}>0$ and $t_{2}-t_{1}>0$, combining with the preference conditions, we can obtain the conditions for each pattern to happen. The results are summarized in the last two columns of Table 1. 


\section{Appendix B Proof of Proposition 1.}

We again illustrate with Case $1 \mathrm{H}$. Solving the first-order conditions for the share of optimal cars in Eq.(11) yields:

$$
M S B_{H}=M C_{a} \text {, i.e., } \Delta u_{H}+M E B_{a H}+M E B_{n H}=M C_{a},
$$

where $\Delta u_{H}, M E B_{a H}$ and $M E B_{n H}$ are derived from Table 2 and Table 3. Substituting them into Eq.(B.1), we obtain

$$
\begin{aligned}
& -\frac{\beta^{\prime} \gamma}{\beta+\gamma} \frac{N}{s}\left(\frac{\alpha^{\prime}}{\alpha}(1-f)+\frac{\gamma^{\prime}}{\gamma} f\right)+\frac{\gamma N}{s}-\frac{\gamma}{\beta+\gamma}\left(\gamma^{\prime} f+\frac{\gamma \alpha^{\prime}}{\alpha}(1-f)\right) \frac{N}{s} \\
& +\frac{\gamma^{2}}{\beta+\gamma}\left(\frac{\gamma^{\prime}}{\gamma}-\frac{\alpha^{\prime}}{\alpha}\right) \frac{(1-f) N}{s}-\frac{\beta^{\prime} \gamma}{\beta+\gamma}\left(\frac{\gamma^{\prime}}{\gamma}-\frac{\alpha^{\prime}}{\alpha}\right) \frac{f N}{s}=M C_{a}-2 \theta_{H} T_{f} .
\end{aligned}
$$

Solving Eq.(B.2) yields the optimal under second-best public provision $f_{1 H}^{p u b}$. On the other hand, the interior equilibrium of Case $1 \mathrm{H}$ in Table 5 is,

$$
\begin{aligned}
f_{1 H}^{M C} & =1-\frac{\alpha \beta^{\prime}\left(\gamma^{\prime}-\gamma\right) N+\alpha(\beta+\gamma)\left(M C_{a}-2 \theta_{H} T_{f}\right) \cdot s}{\left(\alpha \gamma^{\prime}-\alpha^{\prime} \gamma\right)\left(\beta^{\prime}+\gamma\right) N} \\
& =\frac{\left(\alpha \gamma^{\prime}-\alpha^{\prime} \gamma\right)\left(\beta^{\prime}+\gamma\right) N-\alpha \beta^{\prime}\left(\gamma^{\prime}-\gamma\right) N-\alpha(\beta+\gamma)\left(M C_{a}-2 \theta_{H} T_{f}\right) \cdot s}{\left(\alpha \gamma^{\prime}-\alpha^{\prime} \gamma\right)\left(\beta^{\prime}+\gamma\right) N}
\end{aligned}
$$

Combining Eq.(B.2) and Eq.(B.3), after a few steps of calculation, we can get the relationshipship between $f_{1 H}^{p u b}$ and $f_{1 H}^{M C}$,

$$
f_{1 H}^{p u b}=\frac{1}{2} f_{1 H}^{M C}+\frac{\gamma}{2\left(\beta^{\prime}+\gamma\right)}
$$

as expressed in Proposition 1.

Using the same logic, we can obtain the relationship in other cases.

\section{Appendix C The equilibrium share of drivers with autonomous car.}

For presentation purposes, we denote $M C_{a}^{\prime}=M C_{a}-2 \theta_{H} T_{f}$, where the $2 \theta_{H} T_{f}$ is derived from the utility difference. The first column in Table 5 is the range of $\theta_{H} / \theta_{W}$, the second column is the condition of $M C_{a}^{\prime}$ that leads to interior share shown in the third column, and the last two columns show the condition of $M C_{a}^{\prime}$ that leads to the corner solution of 0 and 1 .

\section{Appendix D Proof of Proposition 3.}

We illustrate with Case $1 \mathrm{H}$. The profit of the private monopolist in this case can be expressed as:

$$
\Pi_{1 H}=\left(\Delta u_{H}-M C_{a}\right) f N=\left(\theta_{H} T_{f}-c_{a}+c_{n}-M C_{a}\right) f N
$$




\section{Table 5}

No-toll equilibrium share of autonomous cars under marginal cost provision.

\begin{tabular}{lcccc}
\hline$\theta_{H} / \theta_{W}$ & $M C_{a}^{\prime}{ }^{*}$ & Interior $f^{M C}$ & $M C_{a}^{\prime}$ & Corner $f^{M C}$ \\
\hline \multirow{2}{*}{$\frac{\alpha}{\alpha-\beta}$} & $<\frac{\theta_{H} \cdot \beta^{\prime} \gamma}{\alpha^{\prime} \gamma+\alpha \beta^{\prime}} \frac{N}{s}$ & $1-\frac{\alpha \beta^{\prime}\left(\gamma^{\prime}-\gamma\right) N+\alpha(\beta+\gamma) M C_{a}^{\prime} \cdot s}{\left(\alpha \gamma^{\prime}-\alpha^{\prime} \gamma\right)\left(\beta^{\prime}+\gamma\right) N}$ & $<\frac{\beta^{\prime}\left(\theta_{W}-\theta_{H}\right)}{\beta \gamma} \frac{\delta N}{s}$ & 1 \\
& $>\frac{\theta_{H} \cdot \beta^{\prime} \gamma}{\alpha^{\prime} \gamma+\alpha \beta^{\prime}} \frac{N}{s}$ & $\frac{\alpha^{\prime} \gamma\left(\beta-\beta^{\prime}\right) N-\alpha^{\prime} \cdot(\beta+\gamma) \cdot M C_{a}^{\prime} \cdot s}{\left(\alpha^{\prime} \beta-\alpha \beta^{\prime}\right)\left(\beta^{\prime}+\gamma\right)}$ & $>\frac{\left(\theta_{H}-\theta_{W}\right)}{\beta} \frac{\delta N}{s}$ & 0 \\
$<\frac{\alpha}{\alpha+\gamma}$ & $<\frac{\theta_{H} \cdot \beta \gamma^{\prime}}{\alpha^{\prime} \beta+\alpha \gamma^{\prime}} \frac{N}{s}$ & $1-\frac{\alpha \gamma^{\prime}\left(\gamma-\gamma^{\prime}\right) N+\alpha(\beta+\gamma) M C_{a}^{\prime} \cdot s}{\left(\alpha \beta^{\prime}-\alpha^{\prime} \beta\right)\left(\beta+\gamma^{\prime}\right) N}$ & $<\frac{\gamma^{\prime}\left(\theta_{H}-\theta_{W}\right)}{\beta \gamma} \frac{\delta N}{s}$ & 1 \\
& $>\frac{\theta_{H} \cdot \beta \gamma^{\prime}}{\alpha^{\prime} \beta+\alpha \gamma^{\prime}} \frac{N}{s}$ & $\frac{\alpha^{\prime} \beta\left(\gamma-\gamma^{\prime}\right) N-\alpha^{\prime}(\beta+\gamma) \cdot M C_{a}^{\prime} \cdot s}{\left(\alpha^{\prime} \gamma-\alpha \gamma^{\prime}\right)\left(\beta+\gamma^{\prime}\right) N}$ & $>\frac{\left(\theta_{W}-\theta_{H}\right)}{\gamma} \frac{\delta N}{s}$ & 0 \\
$\left(1, \frac{\alpha}{\alpha-\beta}\right)$ & $<\frac{\left(\theta_{H}-\theta_{W}\right) \delta N}{\beta s}$ & $1-\frac{\alpha \beta^{\prime}\left(\gamma^{\prime}-\gamma\right) N+\alpha(\beta+\gamma) M C_{a}^{\prime} \cdot s}{\left(\alpha \gamma^{\prime}-\alpha^{\prime} \gamma\right)\left(\beta^{\prime}+\gamma\right) N}$ & $<\frac{\beta^{\prime}\left(\theta_{W}-\theta_{H}\right)}{\beta \gamma} \frac{\delta N}{s}$ & 1 \\
& $>\frac{\left(\theta_{H}-\theta_{W}\right) \delta N}{\beta s}$ & $\frac{\theta_{H}(\beta+\gamma)\left(\theta_{H} \delta N-\alpha \cdot M C_{a}^{\prime} \cdot s\right)}{\left(\alpha \beta^{\prime}-\alpha^{\prime} \beta\right)\left(\alpha \gamma^{\prime}-\alpha^{\prime} \gamma\right)}$ & $>\frac{\theta_{H}}{\alpha} \frac{\delta N}{s}$ & 0 \\
$\left(\frac{\alpha}{\alpha+\gamma}, 1\right)$ & $<\frac{\left(\theta_{W}-\theta_{H}\right) \delta N}{\beta s}$ & $1-\frac{\alpha \gamma^{\prime}\left(\gamma-\gamma^{\prime}\right) N+\alpha(\beta+\gamma) M C_{a}^{\prime} \cdot s}{\left(\alpha \beta^{\prime}-\alpha^{\prime} \beta\right)\left(\beta+\gamma^{\prime}\right) N}$ & $<\frac{\gamma^{\prime}\left(\theta_{H}-\theta_{W}\right)}{\beta \gamma} \frac{\delta N}{s}$ & 1 \\
\hline
\end{tabular}

${ }^{*}$ We use $M C_{a}^{\prime}$ to denote $M C_{a}-2 \theta_{H} T_{f}$.

where $c_{a}$ and $c_{n}$ are given in the Case $1 \mathrm{H}$ of Table 2 .

Taking the derivative of $\Pi_{1 H}$ with respect to $f$ yields

$$
\frac{\partial \Pi_{1 H}}{\partial f}=\left(-\frac{\partial c_{a}}{\partial f}+\frac{\partial c_{n}}{\partial f}\right) f N+\left(\theta_{H} T_{f}-c_{a}+c_{n}-M C_{a}\right) N=0 .
$$

From Table 2, we have

$$
\frac{d c_{a}}{d f}=\frac{\beta^{\prime} \gamma}{\beta+\gamma}\left(\frac{\gamma^{\prime}}{\gamma}-\frac{\alpha^{\prime}}{\alpha}\right) \frac{N}{s} ; \quad \frac{d c_{n}}{d f}=-\frac{\gamma^{2}}{\beta+\gamma}\left(\frac{\gamma^{\prime}}{\gamma}-\frac{\alpha^{\prime}}{\alpha}\right) \frac{N}{s} .
$$

Substituting Eq.(D.3) and the travel cost of Case 1H in Table 2 into Eq.(D.2) yields

$$
-\frac{\beta^{\prime} \gamma}{\beta+\gamma}\left(\frac{\gamma^{\prime}}{\gamma}-\frac{\alpha^{\prime}}{\alpha}\right) \frac{f N}{s}-\frac{\gamma^{2}}{\beta+\gamma}\left(\frac{\gamma^{\prime}}{\gamma}-\frac{\alpha^{\prime}}{\alpha}\right) \frac{f N}{s}-\frac{\gamma\left(\beta^{\prime}+\gamma\right)}{\beta+\gamma} \frac{\alpha^{\prime}}{\alpha} \frac{(1-f) N}{s}-\frac{\left(\beta^{\prime}+\gamma\right) \gamma^{\prime}}{\beta+\gamma} \frac{f N}{s}=-\frac{\gamma N}{s}+M C_{a}-2 \theta_{H} T_{f} .
$$

By solving Eq.(D.4) and taking some algebraic manipulations, we can obtain the optimal share under private monopoly provision,

$$
f_{1 H}^{P M}=\frac{\gamma N\left(\alpha^{\prime}\left(\beta^{\prime}+\gamma\right)-\alpha(\beta+\gamma)\right)+\alpha(\beta+\gamma)\left(M C_{a}-2 \theta_{H} T_{f}\right) s}{2\left(\beta^{\prime}+\gamma\right)\left(\alpha^{\prime} \gamma-\alpha \gamma^{\prime}\right) N},
$$

which can be further simplified as

$$
f_{1 H}^{P M}=\frac{1}{2}\left(1-\frac{\alpha \beta^{\prime}\left(\gamma^{\prime}-\gamma\right) N+\alpha(\beta+\gamma)\left(M C_{a}-2 \theta_{H} T_{f}\right) s}{\left(\alpha \gamma^{\prime}-\alpha^{\prime} \gamma\right)\left(\beta^{\prime}+\gamma\right) N}\right) .
$$

Comparing Eq.(D.6) with $f_{1 H}^{M C}$ in Table 5, we have

$$
f_{1 H}^{P M}=\frac{1}{2} f_{1 H}^{M C} .
$$

Similarly, we can prove $f^{P M}=\frac{1}{2} f^{M C}$ is also satisfied in other cases. 


\section{Appendix E Mark-up under second-best public provision.}

As mentioned in Section 5.2, the public provision maximizes social welfare defined as:

$$
\begin{aligned}
S W= & \int_{0}^{N_{a}+N_{n}} D(x) d x+u_{a}\left(N_{a}, N_{n}\right) \cdot N_{a}+u_{n}\left(N_{a}, N_{n}\right) \cdot N_{n}-M C_{a} \cdot N_{a} \\
& -\lambda_{a}\left(D(N)+u_{a}\left(N_{a}, N_{n}\right)-M C_{a}-M U_{a}\right)-\lambda_{n}\left(D(N)+u_{n}\left(N_{a}, N_{n}\right)\right) .
\end{aligned}
$$

The first-order conditions for $N_{a}, N_{n}, M U_{a}, \lambda_{a}$ and $\lambda_{n}$ are:

$$
\begin{aligned}
& \frac{\partial S W}{\partial N_{a}}=D(N)+u_{a}+\frac{\partial u_{a}}{\partial N_{a}} N_{a}+\frac{\partial u_{n}}{\partial N_{a}} N_{n}-M C_{a}-\lambda_{a}\left(D^{\prime}+\frac{\partial u_{a}}{\partial N_{a}}\right)-\lambda_{n}\left(D^{\prime}+\frac{\partial u_{n}}{\partial N_{a}}\right)=0 ; \\
& \frac{\partial S W}{\partial N_{n}}=D(N)+\frac{\partial u_{a}}{\partial N_{n}} N_{a}+u_{n}+\frac{\partial u_{n}}{\partial N_{n}} N_{n}-\lambda_{a}\left(D^{\prime}+\frac{\partial u_{a}}{\partial N_{n}}\right)-\lambda_{n}\left(D^{\prime}+\frac{\partial u_{n}}{\partial N_{n}}\right)=0 ; \\
& \frac{\partial S W}{\partial M U_{a}}=\lambda_{a}=0 ; \\
& \frac{\partial S W}{\partial \lambda_{a}}=-\left(D+u_{a}-M C_{a}-M U_{a}\right)=0 ; \\
& \frac{\partial S W}{\partial \lambda_{n}}=-\left(D+u_{n}\right)=0 .
\end{aligned}
$$

Substituting Eq.(E.5) and Eq.(E.7) into Eq.(E.4) yields

$$
\lambda_{n}=\frac{\frac{\partial u_{a}}{\partial N_{n}} N_{a}+\frac{\partial u_{n}}{\partial N_{n}} N_{n}}{D^{\prime}+\frac{\partial u_{n}}{\partial N_{n}}} .
$$

By substituting Eq.(E.5), Eq.(E.6) and Eq.(E.8) into Eq.(E.3), we can obtain the $M U_{a}$ under second-best public provision, as expressed in Eq.(20). The same logic applies to profit maximization.

\section{References}

Arnott, R., de Palma, A., and Lindsey, R. (1988). Schedule delay and departure time decisions with heterogeneous commuters. Transportation Research Record, 1197:56-67.

Arnott, R., de Palma, A., Lindsey, R., et al. (1990). Economics of a bottleneck. Journal of urban Economics, 27(1):111-130.

Chen, T. D., Kockelman, K. M., and Hanna, J. P. (2016a). Operations of a shared, autonomous, electric vehicle fleet: Implications of vehicle \& charging infrastructure decisions. Transportation Research Part A: Policy and Practice, 94:243-254.

Chen, Z., He, F., Zhang, L., and Yin, Y. (2016b). Optimal deployment of autonomous vehicle lanes with endogenous market penetration. Transportation Research Part C: Emerging Technologies, 72:143156. 
Daganzo, C. F. (1985). The uniqueness of a time-dependent equilibrium distribution of arrivals at a single bottleneck. Transportation science, 19(1):29-37.

de Almeida Correia, G. H. and van Arem, B. (2016). Solving the user optimum privately owned automated vehicles assignment problem (uo-poavap): A model to explore the impacts of self-driving vehicles on urban mobility. Transportation Research Part B: Methodological, 87:64-88.

de Oliveira, Í. R. (2017). Analyzing the performance of distributed conflict resolution among autonomous vehicles. Transportation Research Part B: Methodological, 96:92-112.

Fagnant, D. J. and Kockelman, K. (2015). Preparing a nation for autonomous vehicles: opportunities, barriers and policy recommendations. Transportation Research Part A: Policy and Practice, 77:167181.

Fajardo, D., Au, T.-C., Waller, S., Stone, P., and Yang, D. (2011). Automated intersection control: Performance of future innovation versus current traffic signal control. Transportation Research Record: Journal of the Transportation Research Board, (2259):223-232.

Greenblatt, J. B. and Saxena, S. (2015). Autonomous taxis could greatly reduce greenhouse-gas emissions of us light-duty vehicles. Nature Climate Change, 5(9):860.

Hendrickson, C. and Kocur, G. (1981). Schedule delay and departure time decisions in a deterministic model. Transportation science, 15(1):62-77.

Jamson, A. H., Merat, N., Carsten, O. M., and Lai, F. C. (2013). Behavioural changes in drivers experiencing highly-automated vehicle control in varying traffic conditions. Transportation research part C: emerging technologies, 30:116-125.

Katrakazas, C., Quddus, M., Chen, W.-H., and Deka, L. (2015). Real-time motion planning methods for autonomous on-road driving: State-of-the-art and future research directions. Transportation Research Part C: Emerging Technologies, 60:416-442.

Lamotte, R., de Palma, A., and Geroliminis, N. (2017). On the use of reservation-based autonomous vehicles for demand management. Transportation Research Part B: Methodological, 99:205-227.

Le Vine, S., Zolfaghari, A., and Polak, J. (2015). Autonomous cars: The tension between occupant experience and intersection capacity. Transportation Research Part C: Emerging Technologies, 52:114. 
Levin, M. W. and Boyles, S. D. (2016). A multiclass cell transmission model for shared human and autonomous vehicle roads. Transportation Research Part C: Emerging Technologies, 62:103-116.

Li, Z.-C., Lam, W. H., and Wong, S. (2017). Step tolling in an activity-based bottleneck model. Transportation Research Part B: Methodological, 101:306-334.

Lindsey, R. (2004). Existence, uniqueness, and trip cost function properties of user equilibrium in the bottleneck model with multiple user classes. Transportation science, 38(3):293-314.

Liu, W. (2018). An equilibrium analysis of commuter parking in the era of autonomous vehicles. Transportation Research Part C: Emerging Technologies, 92:191-207.

Liu, Y. and Nie, Y. M. (2011). Morning commute problem considering route choice, user heterogeneity and alternative system optima. Transportation Research Part B: Methodological, 45(4):619-642.

Ma, J., Li, X., Zhou, F., and Hao, W. (2017). Designing optimal autonomous vehicle sharing and reservation systems: A linear programming approach. Transportation Research Part C: Emerging Technologies, 84:124-141.

Mahmassani, H. S. (2016). 50th anniversary invited article - autonomous vehicles and connected vehicle systems: flow and operations considerations. Transportation Science, 50(4):1140-1162.

Mersky, A. C. and Samaras, C. (2016). Fuel economy testing of autonomous vehicles. Transportation Research Part C: Emerging Technologies, 65:31-48.

Newell, G. F. (1987). The morning commute for nonidentical travelers. Transportation Science, $21(2): 74-88$.

Nourinejad, M., Bahrami, S., and Roorda, M. J. (2018). Designing parking facilities for autonomous vehicles. Transportation Research Part B: Methodological, 109:110-127.

Silva, H. E., Lindsey, R., de Palma, A., and Van den Berg, V. A. C. (2016). On the existence and uniqueness of equilibrium in the bottleneck model with atomic users. Transportation Science, $51(3): 863-881$.

Small, K. A. (1982). The scheduling of consumer activities: work trips. The American Economic Review, 72(3):467-479.

Smith, M. J. (1984). The existence of a time-dependent equilibrium distribution of arrivals at a single bottleneck. Transportation science, 18(4):385-394. 
Tseng, Y.-Y. and Verhoef, E. T. (2008). Value of time by time of day: A stated-preference study. Transportation Research Part B: Methodological, 42(7-8):607-618.

van den Berg, V. A. C. and Verhoef, E. T. (2011a). Congestion tolling in the bottleneck model with heterogeneous values of time. Transportation Research Part B: Methodological, 45(1):60-78.

van den Berg, V. A. C. and Verhoef, E. T. (2011b). Winning or losing from dynamic bottleneck congestion pricing?: The distributional effects of road pricing with heterogeneity in values of time and schedule delay. Journal of Public Economics, 95(7):983-992.

van den Berg, V. A. C. and Verhoef, E. T. (2016). Autonomous cars and dynamic bottleneck congestion: The effects on capacity, value of time and preference heterogeneity. Transportation Research Part B: Methodological, 94:43-60.

Varaiya, P. (1993). Smart cars on smart roads: problems of control. IEEE Transactions on automatic control, 38(2):195-207.

Vickrey, W. S. (1969). Congestion theory and transport investment. The American Economic Review, $59(2): 251-260$.

Wadud, Z., MacKenzie, D., and Leiby, P. (2016). Help or hindrance? the travel, energy and carbon impacts of highly automated vehicles. Transportation Research Part A: Policy and Practice, 86:1-18.

Yang, B. and Monterola, C. (2016). Efficient intersection control for minimally guided vehicles: A self-organised and decentralised approach. Transportation Research Part C: Emerging Technologies, $72: 283-305$.

Zakharenko, R. (2016). Self-driving cars will change cities. Regional Science and Urban Economics, $61: 26-37$. 